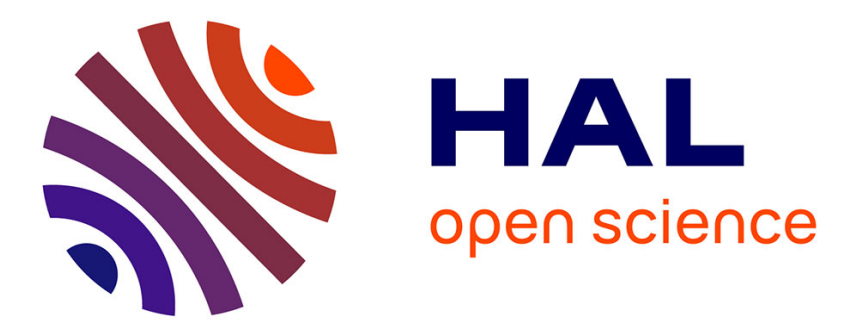

\title{
An explicit time-integrator with singular mass for non-smooth dynamics
}

Jean Di Stasio, David Dureisseix, Gabriel Georges, Anthony Gravouil, Thomas Homolle

\section{- To cite this version:}

Jean Di Stasio, David Dureisseix, Gabriel Georges, Anthony Gravouil, Thomas Homolle. An explicit time-integrator with singular mass for non-smooth dynamics. Computational Mechanics, 2021, 68, pp.97-112. 10.1007/s00466-021-02021-5 . hal-03212569v2

\section{HAL Id: hal-03212569 \\ https://hal.science/hal-03212569v2}

Submitted on 29 Jun 2021

HAL is a multi-disciplinary open access archive for the deposit and dissemination of scientific research documents, whether they are published or not. The documents may come from teaching and research institutions in France or abroad, or from public or private research centers.
L'archive ouverte pluridisciplinaire $\mathbf{H A L}$, est destinée au dépôt et à la diffusion de documents scientifiques de niveau recherche, publiés ou non, émanant des établissements d'enseignement et de recherche français ou étrangers, des laboratoires publics ou privés.

\section{다)(1) $(5$}

Distributed under a Creative Commons Attribution - NonCommercial| 4.0 International 


\title{
An explicit time-integrator with singular mass for non-smooth dynamics
}

\author{
Di Stasio Jean ${ }^{1,2}$, Dureisseix David ${ }^{1}$, Georges Gabriel ${ }^{2}$, Gravouil Anthony ${ }^{1}$, and Homolle \\ Thomas $^{2}$ \\ ${ }^{1}$ Univ Lyon, INSA-Lyon, CNRS UMR5259, LaMCoS, F-69621 Villeurbanne, France \\ ${ }^{2}$ Centre de technologie de Ladoux - Manufacture française de pneumatiques Michelin, \\ Clermont-Ferrand, France
}

Received: 11 January 2021 / Accepted: 12 April 2021 / Published: 28 April 2021

This is a preprint of an article published in Computational Mechanics as Jean Di Stasio, David Dureisseix, Gabriel Georges, Anthony Gravouil, Thomas Homolle An explicit time-integrator with singular mass for non-smooth dynamics. Comput Mech 68:97-112, 2021. The final authenticated version is available online at: https://doi.org/10.1007/ s00466-021-02021-5

Keywords impact; symplectic mechanics; singular mass matrix; unilateral contact; energy conservation

\section{Abstract}

This article addresses the simulation of non-smooth dynamics problems with unilateral contact constraints between rigid and deformable bodies. It proposes a modified CD-Lagrange scheme with a singular mass matrix. This scheme is explicit, and based on a contact condition on velocity. The formulation is designed for a 1D impact problem between deformable and rigid body with unilateral constraint. The singular mass matrix allows to get a conservative energy balance on the discrete system even during nonsmooth events, under the assumption of detecting the release time. This property is new for explicit schemes in non-smooth contact dynamics. An extension is presented then for the $3 \mathrm{D}$ cases. Its implementation is easy, and fully compatible with large deformations or non-linear materials. Indeed it consists only in adding a numerical parameter for each contact node. If the energy conservation is not achieved in $3 \mathrm{D}$, the energy balance is improved thanks to the singular mass matrix.

\section{Introduction}

In transient dynamics simulations of deformable solids, a particular case of interest concerns the simulation of impacts. In mechanical design, these situations may occur in standard configurations like forming in manufacturing process. But above all they are met in accidental scenario of usage with very high loads, like shocks. The numerical simulations are here crucial to predict the mechanical properties and reduce the tests on real structures.

Many numerical schemes are designed for transient dynamics evolutions, and some of them are adapted to deal with impacts (for a review [see 14]). Only few are specially designed to cope with such non-smooth cases, especially for discrete systems in space and time as in [27, 20, 6, 4, 21, 16, 25].

Impact simulations usually require small time steps 
in order to catch the fast phenomena. As the cost of the simulation may increase accordingly, some strategies address this issue by reducing the computational cost of a time-step as explicit schemes [see 5, 2, 29, 9, 15.

The global properties of integration schemes are: efficiency with regard to computational cost; precision; and rate of space-time convergence. An additional key property is the symplectic feature. It gathers conservation of energy, angular and linear momentums. And it is crucial for accurate longtime simulation, especially for non-linear systems [33, 32, 26]. Note that these properties have to be discussed on the discrete problem, in space and time.

Keeping these aspects are a major issue in presence of impacts and contact releases. Some recent advances in this direction were made using the socalled singular mass matrix concept. But they are limited to implicit schemes. The aim of this article is therefore to bring such improvements in explicit. The singular mass matrix is applied on the CD-Lagrange scheme [15], in the case of frictionless contact between a rigid and a deformable body.

This article is organized as follows. First, the state of the art is detailed in section 2 for implicit schemes with singular mass matrix in presence of impacts. Section 3 proposes a design for the CD-Lagrange scheme aiming to take advantage of the singular mass concept. It is built on a dynamic $1 \mathrm{D}$ test problem to detail the different steps in the designing process, and for validation on a reference case. Then, section 4 presents a possible extension to 3D cases, and illustrates the performances of the developed scheme for an impact problem between a deformable solid and a rigid wall. Finally, conclusions and prospects are exposed.

\section{State of the art on singular mass for implicit schemes}

In structural transient dynamics, most of the timeintegration schemes have been designed for unconstrained case. The HHT-Newmark schemes [28, 19, 8] are the most used in this framework. They are stable, and energy conservative or dissipative depending on the choice of scheme parameters [24]. Following the work of Simo and Tarnow 33, 32, variational integrators have been then developed. They are symplectic, meaning that they conserve linear momentum, energy and angular momentum [26]. This conservation property is well suited to non-linear problems especially for running accurate long time simulations.

But the structure of the dynamical problem is deeply changed with contact constraints. The unilateral contact constraint leads to the Signorini's conditions [10]. They state three conditions (sometimes called "non-penetrability conditions"): (i) bodies can not penetrate each other, (ii) contact stresses are normal to the boundary, and (iii) are compressive. With these, the velocity is discontinuous around impact time, and the solution may not be unique. For a clear example on an elastodynamic frictionless contact problem see [22].

The Signorini's conditions are commonly imposed on a semi-discrete system in space by Lagrange multipliers. Indeed this method enforces them exactly. Other effective methods exist for enforcing contact constraints as the penalty, or the Nitsche's method 7. But in the following, we focus on Lagrange multipliers. For more details on discretization by finite elements method and enforcement of Signorini's conditions see for example [22, 14].

On semi-discrete systems with contact enforcement by Lagrange multipliers, the time-integrators like HHT-Newmark lose their properties as the energy conservation, and even sometimes the stability. As shown in 14 on a 1D numerical example, the CrankNicholson scheme presents high frequency oscillations at impact on displacement. They are non-physical, and entirely caused by the numerical time-integration. And above all the energy is highly increased during contact for numerical reasons. This scheme is yet stable and energy conservative on the unconstrained case. The oscillations can be damped by introducing numerical dissipation, but the energy balance is widely degraded.

To overcome these difficulties, Moreau introduces in [27, 20] a framework suited to impact dynamics. The acceleration and contact stresses are defined as 
measures on velocity, which can be then discontinuous. Moreover the contact constraint no more relies on displacement but on velocity. With this velocity contact condition, if a point comes in contact its normal velocity is set to zero (for an impact on a steady rigid body). It is not strictly equivalent to Signorini's conditions on displacement, for a discrete system in space and time. Indeed a slight constant penetration arises at impact and persists during contact. But it tends to zero with space-time convergence. The Moreau's framework and velocity contact condition bring high stability at contact, and a better energetical behavior [13, 6]. The Moreau's condition are sometimes called "persistency condition", due to the null velocity during contact. In 25, Laursen and Chawla use the persistency condition together with the symplectic scheme of Simo and Tarnow. The obtained scheme is symplectic, but the price to pay is numerical oscillations (but bounded) on velocity at contact boundary [14].

In order to recover the uniqueness of the solution with the Signorini's conditions, Paoli and Schatzman [30, 29] introduce an impact law. Howewer if it enables to recover the uniqueness of the solution, the discrete energy behavior is not conservative [14, 13.

In 22 the singular mass method is introduced on elastodynamics contact problems. It combines a recovered uniqueness and a stabilization of contact stresses on semi-discrete system with the Signorini's conditions imposed by Lagrange multipliers. The method relies on zero entries in mass matrix for degrees of freedom (DOF) constrained by contact. According to [22, 31, 12, the solution is unique for elastodynamics problems semi-discrete in space. The singular mass matrix changes indeed the problem structure. The equations of contact DOFs are no more hyperbolic, but elliptic. Indeed the inertia term associated to twice-derivative displacement disappears with the cancellation of the mass entry. The semi-discrete formulation with singular mass matrix presents interesting properties proven in [22, 31]. Firstly the persistency condition is automatically met if the nonpenetrability condition is satisfied. This brings stability into contact. Secondly this persistency condition leads to an energy conservative formulation.

These properties allows to use time-integrators from HHT-Newmark family on the singular semi-discrete formulation, with keeping stability and accurate energy balance. Numerical results demonstrate this in 14 for the HHT-Newmark scheme: with a classical mass matrix the energy blows up and the contact stresses highly oscillates; but with a singular mass matrix, the energy balance is only slightly dissipative and the contact stresses become smooth. Dabaghi et al. 11] presents other interesting results, this time on convergence rate, by comparing classical and singular semi-discrete formulations on 1D numerical tests. For some test cases, the Crank-Nicholson scheme diverges with classical mass matrix but converges with singular one. For others, the singular formulation improves at less the convergence rate. In [22, 11, 12, it is a reduction of oscillations on contact stresses which is observed for Crank-Nicholson, Newmark $(\beta=\gamma=1 / 2)$ and backward Euler schemes. The energy balance is also improved: the energy blow-up disappears for Crank-Nicholson method, and the energy balance is less dissipative for Newmark's $(\beta=\gamma=1 / 2)$ scheme.

This improvement of stability for contact stresses is explained in Krause and Walloth 23. For Newmarklike schemes and consistent mass matrix, the contact stresses equilibrate both inertial terms (from mass of contact nodes) and internal nodal forces. But in the continuous case, the contact boundary has no mass. The contact stress equilibrates then only the internal one. With cancelling the mass of contact nodes, the discrete case gets closer from the continuous one.

Hager et al. in [17] apply the singular mass method to Laursen and Chawla's algorithm [25]. This scheme is already energy conservative. And unlike the HHTNewmark's schemes, it uses the persistency condition instead of the non-penetrability one. But its main drawback is symmetric oscillations for velocities and displacement at the contact boundary due to the numerical integration (see for instance [14). In 23 Krause and Walloth state that these oscillations are due to the incompatibility between persistency condition and energy conservation. If the normal velocity on contact boundary is cancelled, some kinetic energy is lost. The Laursen and Chawla algorithm renders then the velocity always equals to the opposite of its preceding value during contact. However with a sin- 
gular mass matrix, the contact nodes have no inertia and then the oscillations should be reduced. Hager et al. observe in [17] such a numerical behaviour.

There are different ways to get a singular mass matrix. In [22] a global constrained minimization problem is solved under two conditions. Firstly the DOFs along the normal to contact boundary must have a null mass. And secondly the mass redistribution should preserve the total mass, the inertial momentum and the center of gravity of the initial problem. As only DOFs along the normal to contact boundary are massless, the singular mass matrix is parametrized by this normal. This represents an extra computational cost, especially with large displacements where the matrix must be built at each time-step with the updated normal. But some local methods exist with a lower computationnal cost. In 17] the singular mass matrix arises from modified quadrature formulas. They still enforce the previously mentioned constraints for preserving the global mass characteristics of the system. But all DOFs of contact nodes have a null mass instead of only the normal ones. Renard in 31] proposes an other local method with two different Galerkin discretization spaces for velocity and displacement. Hauret in [18, and Tkachuk et al. in 34, embed the singular mass matrix in a variational framework. They use a two and three fields Lagrangian by distinguishing displacement, velocity and linear momentum as independent variables. Finally in [12, the shape functions used to compute the mass matrix are weighted. Depending on the weights, the singular mass matrix has different patterns which have an influence on the discrete solution.

In the present article, we do not address the influence of the pattern of the mass matrix and the method to get it. Indeed, improvements in numerical results are observed whatever the singular mass matrix chosen. In the following, the singular mass method is adapted for the explicit CD-Lagrange scheme. The CD-Lagrange scheme [see 15. is based on central difference method for time-integration, and Lagrange multipliers combined with the Moreau's velocity contact condition. With a consistent mass matrix, a loss of energy is observed at impact for a deformable body [see 13]. Indeed the
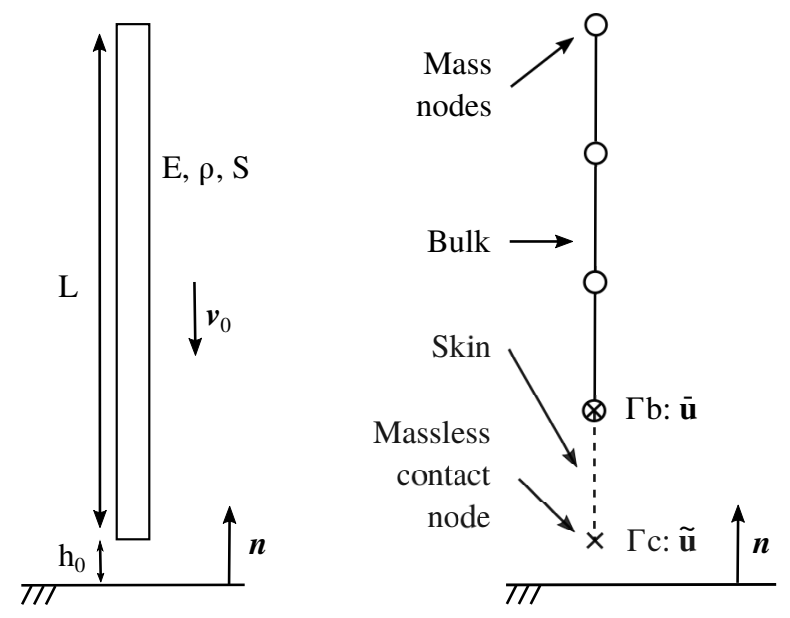

Figure 1: Continuous and discrete in space impacting bar problem

normal velocities of contact nodes are set to zero, cancelling the associated kinetic energy. The goal is then to achieve energy conservation while removing mass of contact nodes and thus the associated inertia.

\section{A CD-Lagrange scheme with singular contact mass for a $1 \mathrm{D}$ case}

\subsection{Scheme introduction on 1D im- pacting bar problem}

We consider the impacting bar problem described in figure 1. This problem is often used as a benchmark in impact mechanics because the analytical solution is known at the contact node [see 14. For numerical results with singular mass matrix, see [11, 12. In [15] and [13, the discrete solution of impacting bar problem is got with the CD-Lagrange scheme, but with a consistent mass matrix.

Linear and homogeneous elasticity is assumed, and the 1D bar impacts a rigid wall with an initial uniform velocity. The spatial discretization is performed with P1 finite elements of equal length. A node corresponds to one degree of freedom (DOF). For more 
details on spatial discretization, see for example [22].

The specificity of the spatial discretization is the massless node at one end of the bar (see figure 1). The mesh is split in the "bulk" and the "skin". The bulk gathers the elements with massive nodes. And the skin is constituted by the element gathering the massless contact node and the bulk node connected. The contact condition is applied on the boundary denoted as $\Gamma_{c}$. And $\Gamma_{b}$ denotes the interface between bulk and skin.

Here the initial mass of contact node is simply cancelled: once the mass matrix has been assembled and lumped, the term corresponding to contact node is set to zero. The mass matrix is then singular. Doing this the total mass is not conserved. Indeed the goal is only to get easily a singular mass matrix. No comparisons will be done between the consistent and the singular mass matrix solutions.

The notations chosen distinguish the bulk and the skin quantities. On the one hand, the bulk problem is a classic finite element discrete system with all massive nodes. It is described by:

- $\mathbf{U}, \dot{\mathbf{U}}, \ddot{\mathbf{U}}$ : displacement, velocity and acceleration vectors gathering DOFs of discrete fields;

- $\mathbf{X}_{\mathbf{0}}$ : initial position of nodes;

- M: mass matrix (consistent, see below);

- $\mathbf{F}^{\text {int }}(\mathbf{U}, \dot{\mathbf{U}})$ : internal forces in the bulk, possibly depending on deformation speed (but elastic in the following);

- $\mathbf{F}^{\text {ext }}$ : external forces;

- $\overline{\mathbf{u}}$ : displacement for DOF on $\Gamma_{b}$;

- $\overline{\mathbf{L}}$ : selects the DOF on $\Gamma_{b}, \overline{\mathbf{u}}=\overline{\mathbf{L}} \mathbf{U}$;

The assemblies of mass and internal force differ. Indeed the global mass matrix $\mathbf{M}^{*}$ is assembled for all elements, and then modified for getting a zero entry for contact DOF. $\mathbf{M}$ is the sub-matrix of $\mathbf{M}^{*}$ corresponding to DOFs of the bulk ( $\mathbf{M}$ is therefore not singular). But for the internal forces $\mathbf{F}\left(\mathbf{U}_{n+1}\right)$, the assembly is performed only on bulk elements. The skin element is not considered.
The spatial semi-discrete system for the bulk is:

$$
\mathbf{M} \ddot{\mathbf{U}}=\mathbf{F}^{\mathrm{ext}}-\mathbf{F}^{\mathrm{int}}(\mathbf{U}, \dot{\mathbf{U}})+\overline{\mathbf{L}}^{t} \widetilde{\mathbf{f}}
$$

where $\widetilde{\mathbf{f}}$ is the force applied by the skin on the bulk. Note here that the system is not submitted to any unilateral contact constraint, $\widetilde{\mathbf{f}}$ is regular being the elastodynamic stress in the skin. $\ddot{\mathbf{U}}$ and $\widetilde{\mathbf{f}}$ are then well defined.

On the other hand, the skin problem has two DOFs: $\overline{\mathbf{u}}$ for the node on $\Gamma_{b}$, and $\widetilde{\mathbf{u}}$ for the contact node on $\Gamma_{c}$. It is described by:

- $\widetilde{\mathbf{u}}$ : displacement of contact massless node;

- $\tilde{\mathbf{x}}_{0}$ : initial position for contact node;

- $\delta \widetilde{\mathbf{u}}=\widetilde{\mathbf{u}}-\overline{\mathbf{u}}$ : difference of displacements for skin nodes, positive if the skin is compressed, negative if stretched;

- $\widetilde{\mathbf{l}}=\left(\overline{\mathbf{x}}_{0}+\overline{\mathbf{u}}\right)-\left(\tilde{\mathbf{x}}_{0}+\widetilde{\mathbf{u}}\right)=\widetilde{\mathbf{l}}_{0}-\delta \widetilde{\mathbf{u}}$ : thickness of skin, $\widetilde{\mathbf{l}}_{0}$ is the initial thickness, with $\overline{\mathbf{x}}_{0}=\overline{\mathbf{L}} \mathbf{X}_{0}$;

- $\widetilde{\mathbf{k}}$ : rigidity of the skin.

Here the choice of skin length and rigidity is made with the initial mesh and rigidity matrix assembled on all elements.

The skin is described by a static behaviour:

$$
\left[\begin{array}{l}
0 \\
0
\end{array}\right]=\left[\begin{array}{c}
-\widetilde{\mathbf{f}} \\
\widetilde{\mathbf{f}}
\end{array}\right]-\left[\begin{array}{cc}
\widetilde{\mathbf{k}} & -\widetilde{\mathbf{k}} \\
-\widetilde{\mathbf{k}} & \widetilde{\mathbf{k}}
\end{array}\right]\left[\begin{array}{c}
\overline{\mathbf{u}} \\
\widetilde{\mathbf{u}}
\end{array}\right]
$$

The skin system (2) added to bulk system (1) forms a global system for all nodes. The node on $\Gamma_{b}$ supports an inertial term from the bulk giving its velocity. But the nodal velocity of $\Gamma_{c}$ is still undetermined according to equations (1) $+(2)$.

Equation (2) condenses in the following expression to give the contact force:

$$
\widetilde{\mathbf{f}}=\widetilde{\mathbf{k}} \delta \widetilde{\mathbf{u}}
$$

Time-integration is performed using the CD-Lagrange scheme [see 15, 13]:

- $h$, the time-step, is kept constant all along the simulation; 
- the time interval $\left[t_{0}, t_{f}\right]$ is discretized with

$$
\left\{\begin{array}{l}
\left\{t_{n}\right\} \mid t_{n}=t_{0}+n * h \\
\left\{t_{n+\frac{1}{2}}\right\} \mid t_{n+\frac{1}{2}}=t_{n}+\frac{h}{2}
\end{array}\right.
$$

- an impulse $\widetilde{\mathbf{r}}_{n+\frac{3}{2}}$ is associated to $\widetilde{\mathbf{f}}$, as its integral over the time interval $\left[t_{n+\frac{1}{2}}, t_{n+\frac{3}{2}}\right]$ :

$$
\widetilde{\mathbf{r}}_{n+\frac{3}{2}}=h \widetilde{\mathbf{f}}_{n+1}=h \widetilde{\mathbf{k}} \delta \widetilde{\mathbf{u}}_{n+1}
$$

Dynamic equation in the bulk (1) are integrated in time as follow [15]:

$$
\begin{aligned}
& \mathbf{U}_{n+1}=\mathbf{U}_{n}+h \dot{\mathbf{U}}_{n+\frac{1}{2}} \\
& \mathbf{M}\left(\dot{\mathbf{U}}_{n+\frac{3}{2}}-\dot{\mathbf{U}}_{n+\frac{1}{2}}\right)= \\
& \quad h\left(\mathbf{F}_{n+1}^{\mathrm{ext}}-\mathbf{F}^{\mathrm{int}}\left(\mathbf{U}_{n+1}, \dot{\mathbf{U}}_{n+\frac{1}{2}}\right)\right) \\
& \quad+\overline{\mathbf{L}}^{t} \widetilde{\mathbf{r}}_{n+\frac{3}{2}}
\end{aligned}
$$

The time integration relation (5) relates two timesteps in explicit fashion. The node velocities are here crucial, and they are determined by the dynamics equation (6) thanks to the inertia term. But no such relation exists for the skin. Note that in order to preserve an explicit integration of internal forces, the term $\mathbf{F}^{\text {int }}\left(\mathbf{U}_{n+1}, \dot{\mathbf{U}}_{n+\frac{1}{2}}\right)$ is computed thanks to the velocity at the previous time-step $\dot{\mathbf{U}}_{n+\frac{1}{2}}$ [see 3]. In the following, the term $\mathbf{F}_{n+1}^{\text {ext }}-\mathbf{F}^{\text {int }}\left(\mathbf{U}_{n+1}, \dot{\mathbf{U}}_{n+\frac{1}{2}}\right)$ is denoted $\mathbf{F}\left(\mathbf{U}_{n+1}\right)$ for shortness but without any loss of generality.

Finally, the contact behaviour acts on the velocity $\widetilde{\mathbf{u}}$ which appears only in time-integration relation:

$$
\widetilde{\mathbf{u}}_{n+1}=\widetilde{\mathbf{u}}_{n}+h \dot{\tilde{\mathbf{u}}}_{n+\frac{1}{2}}
$$

As $\dot{\widetilde{\mathbf{u}}}_{n+\frac{3}{2}}$ is not determined by the dynamics equation, a contact law is proposed. It determines $\dot{\widetilde{\mathbf{u}}}_{n+\frac{3}{2}}$ in order to enforce the contact constraints. This contact law is designed to reproduce the Moreau-Jean's conditions [see 27, 20] which enforce the contact constraints on the velocity. But some adjustments are required as the contact node has no inertia.

Describing the contact law requires:
- $\mathbf{g}_{n+1}$, the gap at the contact node, equal here to $\mathbf{g}_{n+1}=\widetilde{\mathbf{x}}_{0}+\widetilde{\mathbf{u}}_{n+1}$;

- $\dot{\mathbf{U}}_{n+\frac{3}{2}}^{\text {free }}$, the free velocity of the bulk : i.e. the bulk velocity without skin action.

Equation (6) without contact force gives $\dot{\mathbf{U}}_{n+\frac{3}{2}}^{\text {free }}$ as:

$$
\dot{\mathbf{U}}_{n+\frac{3}{2}}^{\text {free }}=\dot{\mathbf{U}}_{n+\frac{1}{2}}+h \mathbf{M}^{-1} \mathbf{F}\left(\mathbf{U}_{\mathbf{n}+\mathbf{1}}\right)
$$

and for the common node to bulk and skin:

$$
\dot{\overline{\mathbf{u}}}_{n+\frac{3}{2}}^{\text {free }}=\overline{\mathbf{L}}_{n+\frac{3}{2}}^{\text {free }}
$$

The proposed velocity contact law is:

$$
\text { If } \mathbf{g}_{n+1}>0, \quad \dot{\widetilde{\mathbf{u}}}_{n+\frac{3}{2}}=\dot{\mathbf{u}}_{n+\frac{3}{2}}^{\text {free }}
$$

Else $\mathbf{g}_{n+1} \leqslant 0$,

$$
\left\{\begin{aligned}
\text { If } \widetilde{\mathbf{r}}_{n+\frac{3}{2}} \geqslant 0, & \dot{\widetilde{\mathbf{u}}}_{n+\frac{3}{2}}=0 \\
\text { Else } \widetilde{\mathbf{r}}_{n+\frac{3}{2}}<0, & \dot{\widetilde{\mathbf{u}}}_{n+\frac{3}{2}}=\left(\dot{\mathbf{u}}_{n+\frac{3}{2}}^{\text {free }}\right)^{+}
\end{aligned}\right.
$$

where $(\bullet)^{+}$returns the positive part.

The main idea herein is to relate the velocity $\dot{\widetilde{\mathbf{u}}}$ of $\Gamma_{c}$ and the velocity $\dot{\overline{\mathbf{u}}}$ of $\Gamma_{b}$. This law concerns two main situations:

- equation (10) describes the free-of-contact state, where the contact node is not in contact;

- and equation (11) describes the active contact state, where the contact node velocity depends on the skin force.

$\dot{\widetilde{\mathbf{u}}}_{n+\frac{3}{2}}$ is always set with the free velocity of the closest node on the bulk. In free-of-contact state, $\dot{\mathbf{u}}_{n+\frac{3}{2}}$ and $\dot{\widetilde{\mathbf{u}}}_{n+\frac{3}{2}}$ are equal only if the skin is at rest $\left(\delta \widetilde{\mathbf{u}}_{n+1}=0\right.$ and then $\widetilde{\mathbf{r}}_{n+\frac{3}{2}}=0$ ). For active contact state, three cases emerge as described in Table 1.

At impact, the skin is considered in its initial rest state. In this particular impacting bar problem this is true. Otherwise the skin tends to relax into rest state during a free-of-contact phase. 


\begin{tabular}{|c|c|c|}
\hline Impact & $\begin{array}{l}\mathbf{g}_{n+1} \leqslant 0 \\
\widetilde{\mathbf{r}}_{n+\frac{3}{2}}=0 \\
\dot{\widetilde{\mathbf{u}}}_{n+\frac{3}{2}}=0\end{array}$ & $\begin{array}{l}\text { The skin is at initial } \\
\text { length, the contact node is } \\
\text { stopped. }\end{array}$ \\
\hline Contact & $\begin{array}{l}\mathbf{g}_{n+1} \leqslant 0 \\
\widetilde{\mathbf{r}}_{n+\frac{3}{2}}>0 \\
\dot{\widetilde{\mathbf{u}}}_{n+\frac{3}{2}}=0\end{array}$ & $\begin{array}{l}\text { The skin is compressed, } \\
\text { the contact node does not } \\
\text { move. }\end{array}$ \\
\hline Release & $\begin{array}{l}\mathbf{g}_{n+1} \leqslant 0 \\
\widetilde{\mathbf{r}}_{n+\frac{3}{2}} \leqslant 0 \\
\dot{\widetilde{\mathbf{u}}}_{n+\frac{3}{2}} \geqslant 0\end{array}$ & $\begin{array}{l}\text { The skin is at rest or } \\
\text { stretched, contact node } \\
\text { moves only to release con- } \\
\text { tact. }\end{array}$ \\
\hline
\end{tabular}

Table 1: Gap, impulse and contact node velocity for contact case

In summary, the discrete system is described by the following equations:

$$
\begin{aligned}
& \mathbf{U}_{n+1}=\mathbf{U}_{n}+h \dot{\mathbf{U}}_{n+\frac{1}{2}} \\
& \mathbf{M}\left(\dot{\mathbf{U}}_{n+\frac{3}{2}}-\dot{\mathbf{U}}_{n+\frac{1}{2}}\right)=h \mathbf{F}\left(\mathbf{U}_{n+1}\right)+\overline{\mathbf{L}}^{t} \widetilde{\mathbf{r}}_{n+\frac{3}{2}} \\
& \widetilde{\mathbf{u}}_{n+1}=\widetilde{\mathbf{u}}_{n}+h \dot{\widetilde{\mathbf{u}}}_{n+\frac{1}{2}} \\
& \left\{\begin{array}{c}
\widetilde{\mathbf{r}}_{n+\frac{3}{2}}=h \widetilde{\mathbf{k}} \delta \widetilde{\mathbf{u}}\left(\overline{\mathbf{u}}_{n+1}, \widetilde{\mathbf{u}}_{n+1}\right) \\
+ \text { Velocity contact law: 10, } 11
\end{array}\right.
\end{aligned}
$$

where the unknowns are:

- the displacements $\mathbf{U}_{n+1}, \widetilde{\mathbf{u}}_{n+1}$ directly computed from (12) and (14);

- the velocities $\dot{\mathbf{U}}_{n+\frac{3}{2}}, \dot{\widetilde{\mathbf{u}}}_{n+\frac{3}{2}}$ get from 13 and (15).

The scheme steps are described in algorithm 1. It is explicit because no system solving is needed. Indeed the update of displacement is direct, and $\dot{\mathbf{U}}_{n+\frac{3}{2}}$ comes from a system with a lumped mass matrix. This singular contact mass variant of the CD-Lagrange preserves the suitable properties for impact problems of the one with a consistent mass [see 13]. As the velocity contact law is very close from the Moreau-Jean's conditions (see the appendix 5 for more details), both schemes present a very stable contact with a slight residual penetration at $\Gamma_{c}$.

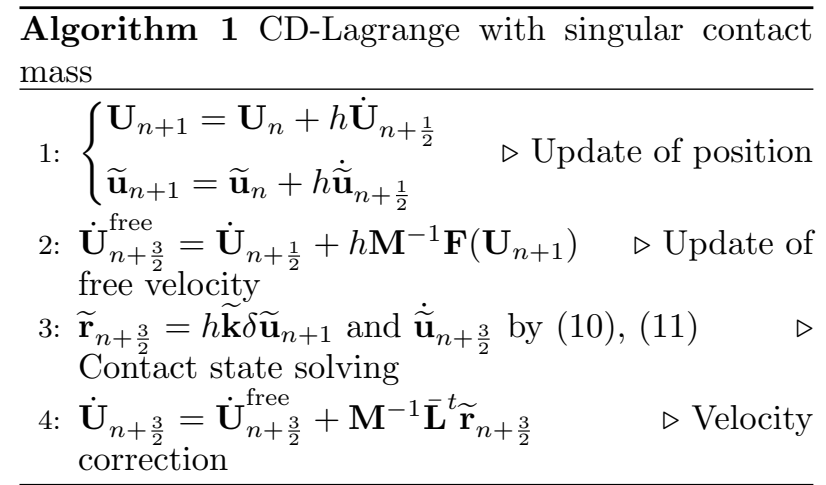

The following energy balance highlights some interesting properties of this scheme. The balance is done between $t_{n+\frac{1}{2}}$ and $t_{n+\frac{3}{2}}$ in order to eliminate the complementary term (see 15, 24 for the balance between $t_{n+1}$ and $t_{n}$ ). The energy balance for the bulk is:

$$
\Delta E_{k}=\Delta \bar{W}_{\mathrm{int}}+\Delta W_{\mathrm{skin}}
$$

With:

$$
\begin{aligned}
& \Delta E_{k}=\left[\frac{1}{2} \dot{\mathbf{U}}^{t} \mathbf{M} \dot{\mathbf{U}}\right]_{n+\frac{1}{2}}^{n+\frac{3}{2}} \\
& \Delta \bar{W}_{\text {int }}=\frac{1}{2} h\left(\dot{\mathbf{U}}_{n+\frac{3}{2}}+\dot{\mathbf{U}}_{n+\frac{1}{2}}\right)^{t} \mathbf{F}\left(\mathbf{U}_{n+1}\right) \\
& \Delta W_{\text {skin }}=\frac{1}{2}\left(\dot{\mathbf{u}}_{n+\frac{3}{2}}+\dot{\overline{\mathbf{u}}}_{n+\frac{1}{2}}\right) \widetilde{\mathbf{r}}_{n+\frac{3}{2}}
\end{aligned}
$$

$\Delta E_{k}$ is the variation of kinetic energy. As the kinetic energy relies on mass, only the bulk is concerned. $\Delta \bar{W}_{\text {int }}$ is the work of internal forces in the bulk. $\Delta W_{\text {skin }}$ is the work on $\Gamma_{b}$ due to the skin, which can be decomposed into:

$$
\Delta W_{\text {skin }}=\Delta \widetilde{W}_{\mathrm{int}}+\Delta W_{c}
$$

With:

$$
\begin{aligned}
& \Delta \widetilde{W}_{\mathrm{int}}=-\frac{1}{2}\left(\delta \dot{\tilde{\mathbf{u}}}_{n+\frac{3}{2}}+\delta \dot{\tilde{\mathbf{u}}}_{n+\frac{1}{2}}\right) \widetilde{\mathbf{r}}_{n+\frac{3}{2}} \\
& \Delta W_{c}=\frac{1}{2}\left(\dot{\tilde{\mathbf{u}}}_{n+\frac{3}{2}}+\dot{\tilde{\mathbf{u}}}_{n+\frac{1}{2}}\right) \widetilde{\mathbf{r}}_{n+\frac{3}{2}}
\end{aligned}
$$

$\Delta \widetilde{W}_{\text {int }}$ is the work of internal forces in the skin, $\Delta W_{c}$ is the work of contact forces. $\delta \dot{\widetilde{\mathbf{u}}}=\dot{\widetilde{\mathbf{u}}}-\dot{\overline{\mathbf{u}}}$ is the 


\begin{tabular}{ll}
\hline Parameters & Values \\
\hline density $\rho$ & $7.85 \times 10^{3} \mathrm{~kg} \cdot \mathrm{m}^{-3}$ \\
Young modulus $E$ & $2.10 \times 10^{11} \mathrm{~Pa}$ \\
beam section $S$ & $6.45 \times 10^{-4} \mathrm{~m}^{2}$ \\
beam length $L$ & $2.54 \times 10^{-1} \mathrm{~m}$ \\
initial velocity $v_{0}$ & $5 \mathrm{~m} . \mathrm{s}^{-1}$ \\
\hline number of elements $N$ & 50 \\
critical time step $d t_{\mathrm{CFL}}$ & $9.82 \times 10^{-7} \mathrm{~s}$ \\
time step $h$ & $8.84 \times 10^{-7} \mathrm{~s}$ \\
\hline
\end{tabular}

Table 2: Numerical values of the 1D test case

deformation speed of skin. The energy balance for bulk and skin is then:

$$
\Delta E_{k}=\Delta \bar{W}_{\mathrm{int}}+\Delta \widetilde{W}_{\mathrm{int}}+\Delta W_{c}
$$

The energy conservation depends on the term $\Delta W_{c}$, that equals zero when:

- $\widetilde{\mathbf{r}}_{n+\frac{3}{2}}=0 \Leftrightarrow \delta \widetilde{\mathbf{u}}_{n+1}=0$ : the skin is in rest state;

- $\left(\dot{\widetilde{\mathbf{u}}}_{n+\frac{3}{2}}+\dot{\widetilde{\mathbf{u}}}_{n+\frac{1}{2}}\right)=0$ : the contact is active for previous and current time-step.

On the impacting bar problem, the skin develops an energy only at release time-step and time-steps after. The release is detected when $\delta \widetilde{\mathbf{u}} \leqslant 0$, meaning that the skin passes from a compressed state during contact to a stretched one at release. During the timesteps after release, the skin goes back to its rest state and so $\delta \widetilde{\mathbf{u}} \neq 0$. The corresponding work is not null and not necessary dissipative. But if the skin does not reach a stretched state, this work is null. To do that, $\delta \widetilde{\mathbf{u}}$ must be equal to zero at release.

This energy balance confirms the idea that the massless contact node solves the loss of energy at impact. Indeed $\Delta W_{c}$ is null at impact. If the release is not conservative, this work can be cancelled. The singular mass matrix makes then possible a conservative energy balance.

\subsection{Numerical results}

The impacting beam described in Figure 1 is set with the values of Table 2. The CFL time-step is $d t_{\mathrm{CFL}}=$ $2\left(\lambda_{\max }\right)^{-1 / 2}$, with $\lambda_{\max }$ the maximum eigenvalue of

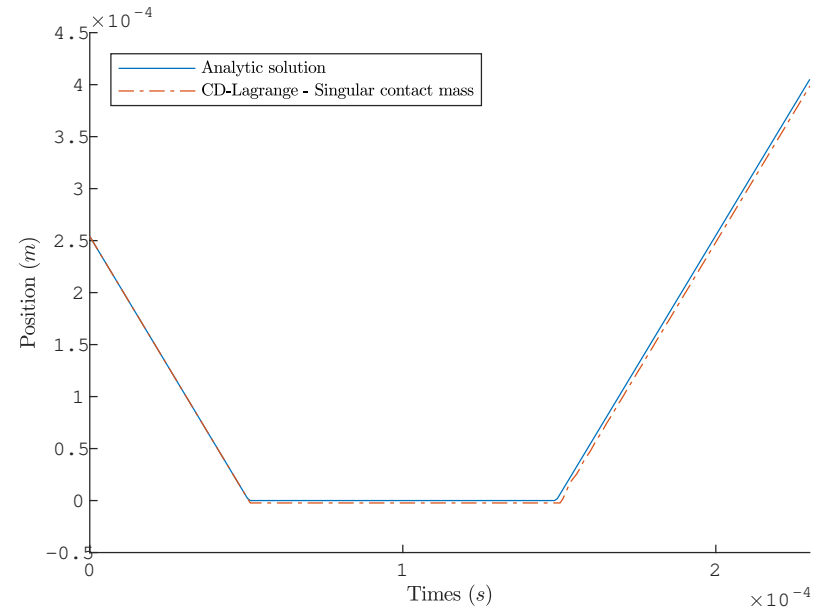

Figure 2: Contact node position

$\mathbf{M}^{-1} \mathbf{K}$ (see [2]). We choose a constant time-step $h=$ $0.9 \times d t_{\mathrm{CFL}}$.

The following charts compare the discrete solution to the analytic one at the contact node.

Figure 2 depicts the position for analytical solution and the discrete one. The main difference between both is the residual penetration during contact for the discrete solution. For velocity on figure 3 , the discrete solution presents spurious oscillations after release. They are present too on the classical CDLagrange [see 13, due to the spatial discretization. On figure 4 the discrete impulse oscillates after impact before stabilizing at a constant value close to the analytic one. At release, the skin impulse reaches a negative minimum before coming back to null value in few time-steps. The negative minimum corresponds to the discrete time where release is detected. The figure 5 shows the global energy transfers. The internal energy on this graph is the sum of bulk and skin internal energies; and the system energy is the sum of conservative terms: $E_{\text {sys }}=E_{k}+E_{\text {int }}+\widetilde{E}_{\text {int }}$. The energetic behaviour is confirmed: the impact is conservative; during contact, the energy is transferred between kinetic and internal ones; and at release, the contact work decreases the system energy when the skin comes back to its rest state.

If the release time matches a discrete time, the skin 


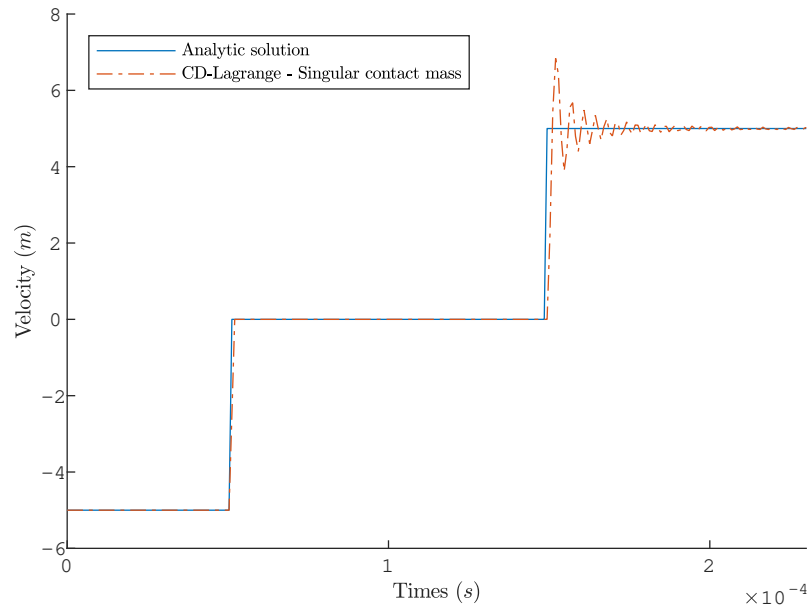

Figure 3: Contact node velocity

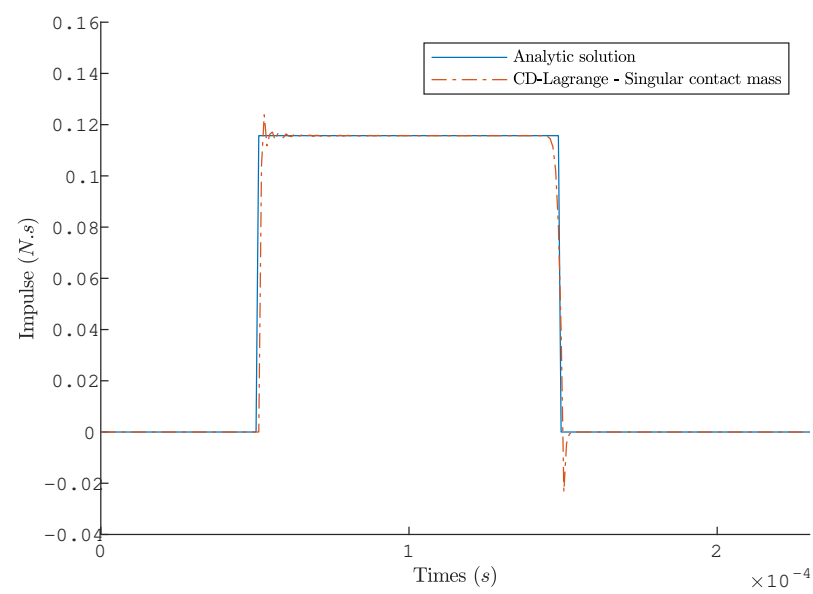

Figure 4: Skin impulse

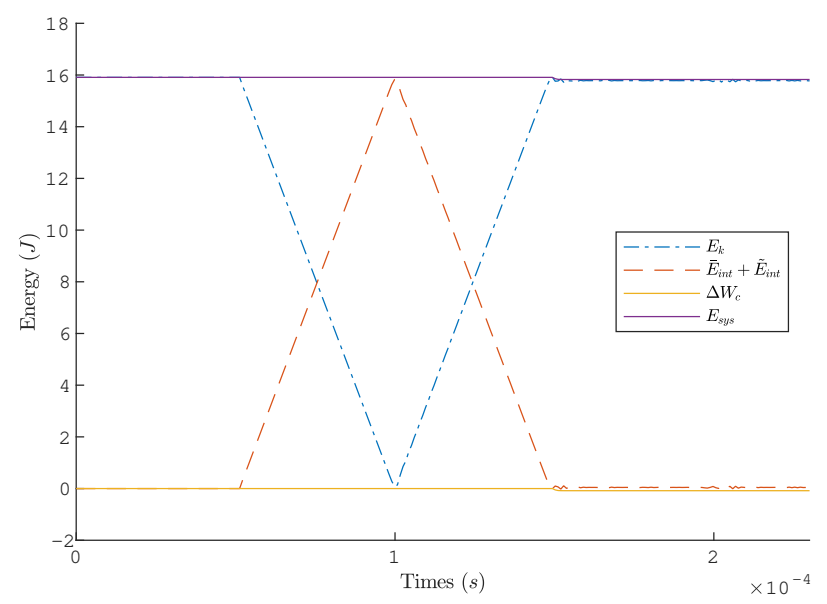

Figure 5: Global energy balance between $\left[t_{n+\frac{1}{2}}, t_{n+\frac{3}{2}}\right]$

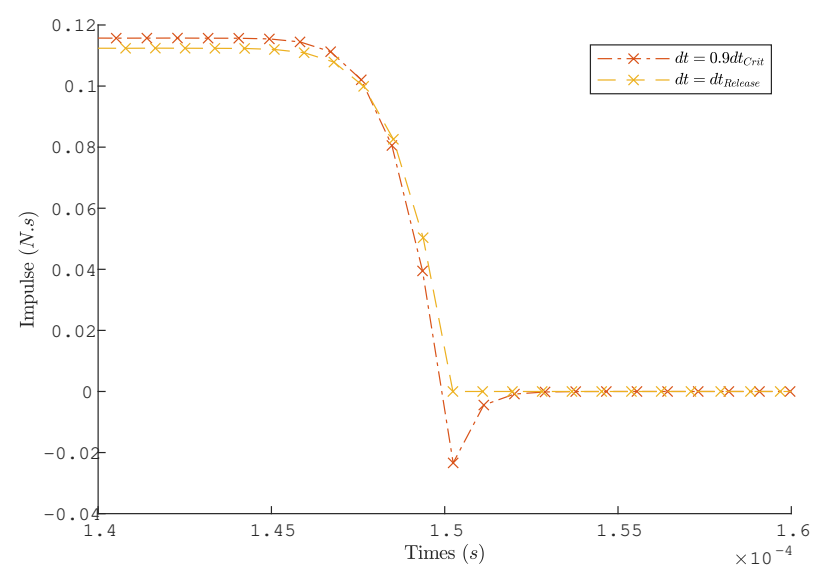

Figure 6: Skin impulse - Release on a discrete time 


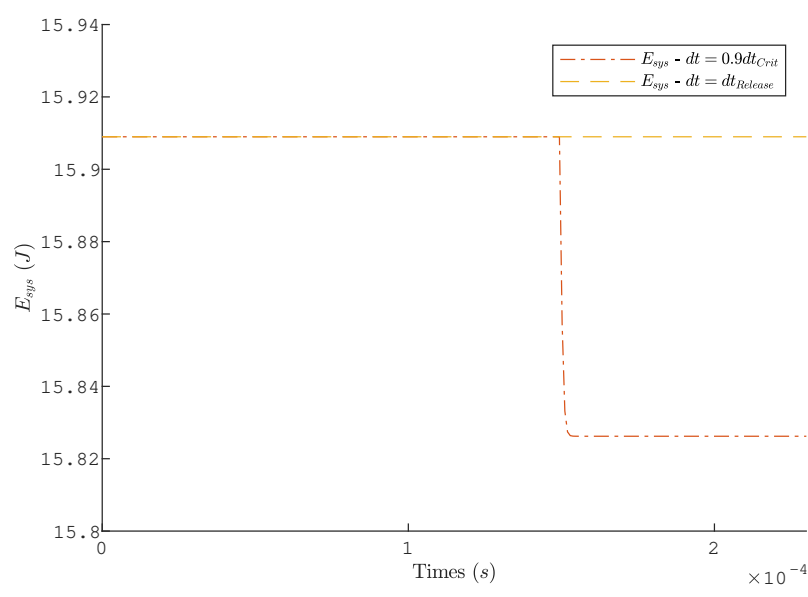

Figure 7: Comparison of system energy

does not reach a stretched state. Thus the skin impulse is not negative after release and does not reduce the system energy. The figures 6 and 7 illustrate this. The time step is chosen in order that a discrete time corresponds exactly to the analytic release time (with keeping the time-step constant during the simulation). $h_{\text {Release }}$ names this time-step, and $h$ the previous one. The impulse for $h_{\text {Release }}$ on figure 6 stays positive (equal to zero at release). The system energy is then constant on figure 7 for $h_{\text {Release }}$, whereas it decreases after release for $h$. Note that the goal of figures 6 and 7 is only to illustrate the energy conservation.

These numerical results confirm that the singular contact mass brings the possibility of a conservative energy balance without degrading the other performances. Even if the conservation of energy is conditioned to a release time matching a discrete time, it is a major advance in explicit schemes for impact between deformable bodies.

\subsection{Choice of skin rigidity and length}

The choice of skin initial length and rigidity is a numerical parameter of the scheme. For the previous impacting bar problem, the skin element keeps the rigidity and length of the problem with a consistent mass. But the same kind of problem is obtained by adding a massless node at the contact boundary in order to form a skin. The skin rigidity and initial length have then to be chosen. In order to lead such a choice, the influence of skin rigidity and length is analysed on the discrete solution for the 1D impacting bar problem.

The initial length of skin has no influence on the shape of discrete solution. Indeed $\widetilde{\mathbf{l}}_{0}$ does not appear in the equations. The skin force, $\widetilde{\mathbf{f}}=\widetilde{\mathbf{k}} \delta \widetilde{\mathbf{u}}$, depends only on $\delta \widetilde{\mathbf{u}}$ : the difference between the displacements of $\Gamma_{c}$ and $\Gamma_{b}$. The initial skin length ensures only that the skin keeps a positive length. Indeed as $\widetilde{\mathbf{l}}=\widetilde{\mathbf{l}}_{0}-\delta \widetilde{\mathbf{u}}$, if $\delta \widetilde{\mathbf{u}}>\widetilde{\mathbf{l}}_{0}$ the skin has a negative length. But the behaviour of $\widetilde{\mathbf{f}}$ stays valid, even when $\widetilde{\mathbf{l}}<0$. $\widetilde{\mathbf{l}}_{0}$ is only a numerical parameter which gives a physical interpretation to the skin. It is not necessary to the formulation.

But as the gap is here computed between $\Gamma_{c}$ and the rigid body, it depends on $\widetilde{\mathbf{l}}_{0}$. A change in $\widetilde{\mathbf{l}}_{0}$ modifies the discrete impact time: earlier than the analytical one with a larger $\widetilde{\mathbf{l}}_{0}$, and later with a smaller one. It raises the question of where the gap should be estimated. This point is discussed later on the 3D case.

The influence of skin rigidity is far more crucial. First, it changes the stable time-step. Here the element rigidity is constant over the beam, and denoted $\widetilde{\mathbf{k}}_{\text {nominal }}$. If $\widetilde{\mathbf{k}}>\widetilde{\mathbf{k}}_{\text {nominal }}$ the time-step must be decreased to keep the time-integration stability. And this is not acceptable for the computational cost. A modification of $\widetilde{\mathbf{k}}$ changes also slightly the shape of the discrete solution, as shown in figures 8, 9, 10 and 11. Here several discrete solutions are computed for several $\widetilde{\mathbf{k}}$ between $0.1 \times \widetilde{\mathbf{k}}_{\text {nominal }}$ and $\widetilde{\mathbf{k}}_{\text {nominal }}$, keeping the same time-step as before.

A modification of $\widetilde{\mathbf{k}}$ causes no changes in the global shape for the position of contact node (figure 8). But the release is smoother for velocities when $\widetilde{\mathbf{k}}$ decrease, with less oscillations for contact node (figure 9). Indeed $\widetilde{\mathbf{k}}$ is directly the stiffness of skin element. The same pattern appears on skin impulses (figure 10) when $\widetilde{\mathbf{k}}$ decreases. On figure 10 the contact impulse reaches the same maximum value whatever the $\widetilde{\mathbf{k}}$ value. Indeed the force applied by the bulk on the skin does not change. The consequence on $\delta \widetilde{\mathbf{u}}$ 


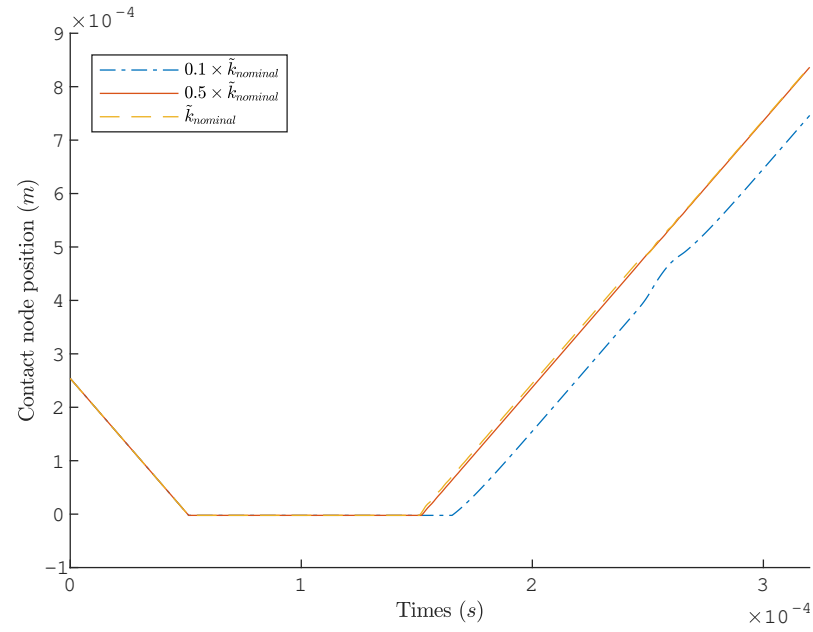

Figure 8: Influence of $\widetilde{\mathbf{k}}$ on contact node position

is shown on figure 11 the maximum value increases when $\widetilde{\mathbf{k}}$ decreases to keep $\widetilde{\mathbf{f}}$ constant.

An interesting property of $\widetilde{\mathbf{k}}$ parameter is that it modifies the time of release (see figure 8). The energy loss at release is related to $\|\delta \widetilde{\mathbf{u}}\|$. Further is the release time from a discrete time, greater is $\|\delta \widetilde{\mathbf{u}}\|$ and greater is the energy loss. Adjusting $\widetilde{\mathbf{k}}$ is then equivalent to adjust the time-step: it can match the release and a discrete time, making the scheme conservative or at less improving the energy balance.

In brief, $\widetilde{\mathbf{k}}$ influences only slightly the discrete solution both on the discrete release time and the skin response. Indeed it is more a numerical parameter, than a physical one. It gives a flexibility for the choice of skin parameter which is useful for extending the scheme to 3D problems. Moreover it does not influence the convergence rate.

The space-time convergence is checked using a mesh refinement. Increasing the number of nodes in bar decreases the element length (constant over the bar). For each mesh, the time-step is set at $90 \%$ of critical time-step. The following Hausdorff measure is used to evaluate the error [see 1]:

$$
e=\frac{1}{2} h \sum_{i \in[1, n]}\left|f_{i}-f\left(t_{i}\right)\right|
$$

where:

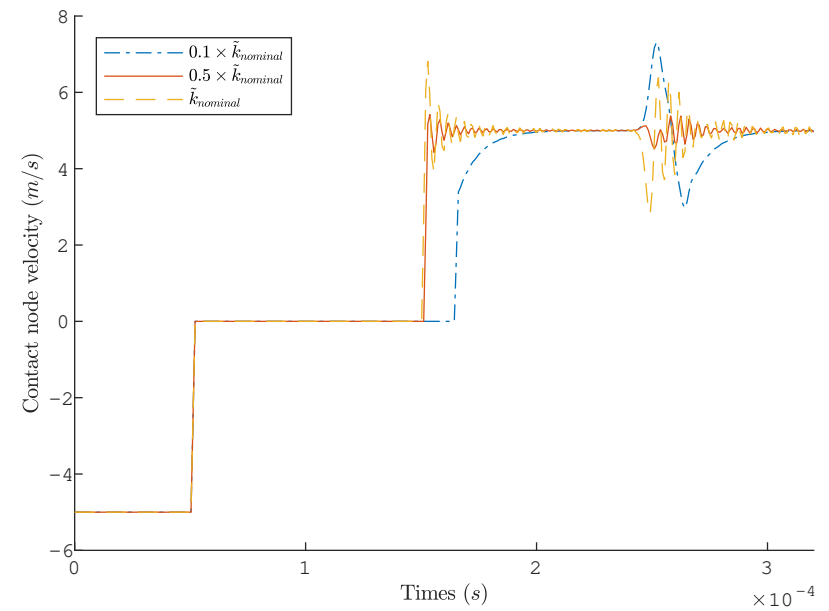

Figure 9: Influence of $\widetilde{\mathbf{k}}$ on contact node velocity

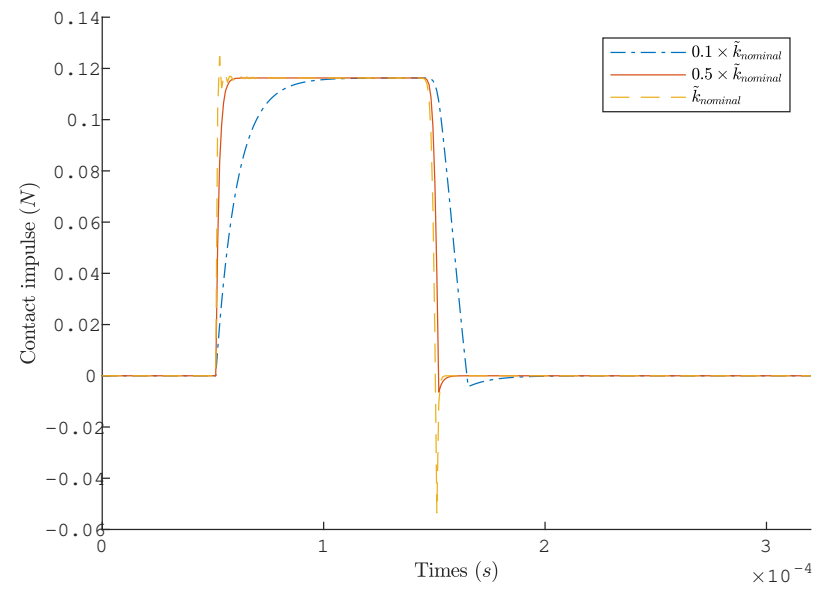

Figure 10: Influence of $\widetilde{\mathbf{k}}$ on skin impulse 


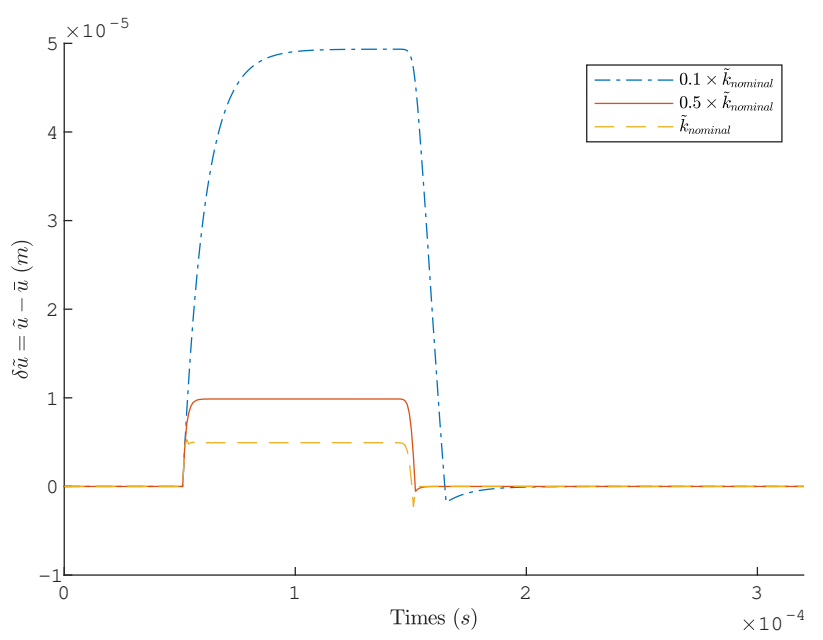

Figure 11: Influence of $\widetilde{\mathbf{k}}$ on $\delta \widetilde{\mathbf{u}}$

- $n$ is the number of discrete times in interval $\left[t_{0}, t_{f}\right]$

- $f_{i}$ is the discrete solution at time $t_{i}$, here at contact node;

- $f\left(t_{i}\right)$ is the analytical solution at time $t_{i}$, here at contact node.

The norm 24 evaluates the distance between the point graph from discrete solution and the continuous graph from analytical solution.

The figures 12 and 13 show no differences in convergence rate for several $\widetilde{\mathbf{k}}$, both on contact node position and contact impulse. The space-time convergence rate is order 1 . This rate of convergence is common for schemes using a contact constraint on velocity. See for example [1, 6 for implicit schemes, or [15, 13. for CD-Lagrange with consistent mass matrix.

\subsection{Conclusions on 1D case}

The CD-Lagrange scheme with singular contact mass gathers the advantages of: an explicit scheme for time integration; a contact constraint at velocity level for stabilising the contact; and a massless contact node for improving the energy balance. The massless contact node brings a conservative impact by cancelling

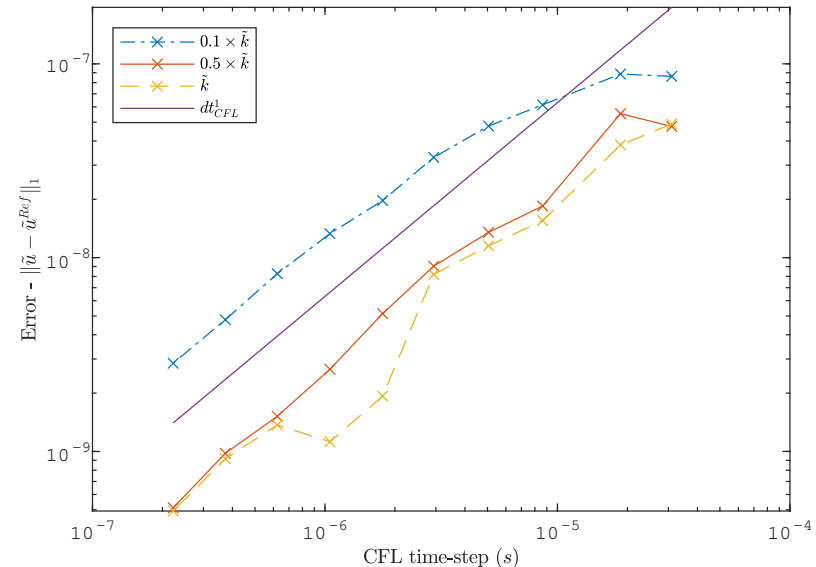

Figure 12: Space-time convergence for contact node position

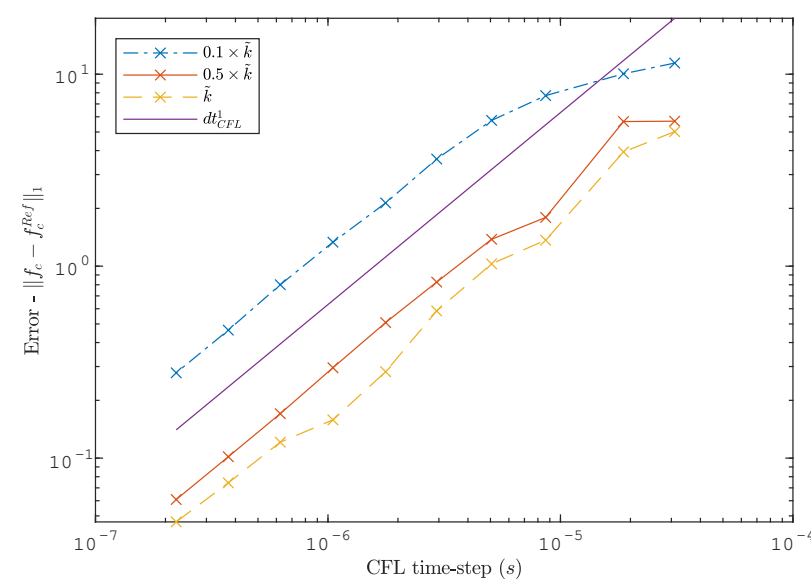

Figure 13: Space-time convergence for skin force 
the inertia of contact boundary. The drawback is that the contact velocity is no more determined by the dynamic. This is overcome by a contact law based on skin stress, which enforces a persistency like contact condition. With the massless contact node, the skin rigidity becomes a numerical parameter which allows to adjust the release time and the skin response. Indeed it does not change the solution at space-time convergence. The major result is a possible conservative energy balance, when the release time matches a discrete time.

Some explicit schemes present a really good energy behavior for impact on deformable bodies, as the Cirak and West's DCR algorithm [9], the pinball algorithm [2], the Paoli-Schatzman's scheme [30, 29] or the CD-Lagrange scheme 15. But as far as we known, no one achieves energy conservation for deformable bodies in non-smooth dynamics. The only conservative explicit schemes on discrete system reported in literature concern rigid bodies as the CDLagrange [15].

In $1 \mathrm{D}$ it is quite simple to adjust $\tilde{\mathbf{k}}$ or the time-step to match the release time with a discrete time. But for a multiple DOF contact, it becomes difficult. As a consequence the goal of the following section is not to achieve energy conservation for multiple DOFs contact. The 1D singular mass CD-Lagrange is extended to a $3 \mathrm{D}$ case for improving the energy balance.

\section{An extension to 3D case: normal elements on contact zone}

\subsection{Adaptation of the 1D formulation to a $3 \mathrm{D}$ case}

For the impacting bar problem, the direction of the beam can be seen as the local normal to contact boundary. With this interpretation, the skin is a 1D normal element added on $\Gamma_{b}$ along the contact normal. This interpretation leads to a quite straightforward 3D extension of the preceding 1D scheme.

Figure 14 represents the contact boundary of a 3D semi-discrete problem in space. This contact bound-

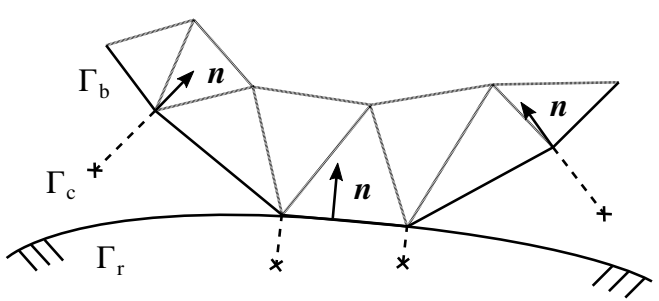

Figure 14: 3D contact problem with normal massless skin

ary $\Gamma_{b}$ impacts a rigid motionless frontier $\Gamma_{r}$ (analytically described). The contact is unilateral, no friction is considered. The problem is discretized in space with P1 finite elements to have DOFs localized on nodes especially on $\Gamma_{b}$.

In order to meet the contact constraint, a "skin" is added on $\Gamma_{b}$. Each node on $\Gamma_{b}$ gets a $1 \mathrm{D}$ normal element with a massless node outward (along $\boldsymbol{- n}$ ). The normal $\boldsymbol{n}$ is the inner normal to $\Gamma_{b}$. When contact happens, $\boldsymbol{n}$ is matching the outer normal of $\Gamma_{r}$. Each normal element is characterized by an artificial rigidity, which is now totally a numerical parameter. It is the extension of the previously $1 \mathrm{D}$ massless skin for $3 \mathrm{D}$ meshes. The persistency condition is, as in 1D, applied on massless nodes.

The "bulk" refers to initial mesh, without added normal skin. It is composed by nodes with three DOFs corresponding to the three components of the displacement field. The discretization is the classical finite element one, with a mass entry for each node. The material law is not necessary elastic; indeed the following formulation does not depend on it. This bulk problem is described by:

- $\mathbf{U}, \dot{\mathbf{U}}, \ddot{\mathbf{U}}$ : vector of DOFs for displacements, velocities and accelerations of bulk nodes, $\mathbf{X}_{0}$ gathers the initial position;

- $\mathbf{M}, \mathbf{F}^{\text {ext }}, \mathbf{F}^{\text {int }}(\mathbf{U}, \dot{\mathbf{U}})$ : lumped mass matrix, external forces, and internal ones depending possibly on velocity;

- $\overline{\mathbf{L}}$ : projection operator on each node of $\Gamma_{b}$ along $n$.

The equation for the semi-discrete problem in 
space is:

$$
\mathbf{M} \ddot{\mathbf{U}}=\mathbf{F}^{\mathrm{ext}}-\mathbf{F}^{\mathrm{int}}(\mathbf{U}, \dot{\mathbf{U}})+\overline{\mathbf{L}}^{t} \widetilde{\mathbf{f}}
$$

$\widetilde{\mathbf{f}}$ is a vector gathering each force applied by normal elements on the bulk. With these notations, the Equation 25 is similar to 1D case.

All normal quantities are gathered in vectors whose size is the number of nodes in $\Gamma_{b}$. For all nodes in $\Gamma_{b}$, identified with subscript $i$, the following normal quantities describe the skin problem:

- $(\widetilde{\mathbf{k}})_{i}$ : artificial rigidity of normal element;

- $(\widetilde{\mathbf{u}})_{i}$ : displacement of a massless node;

- $(\overline{\mathbf{u}})_{i}$ : normal displacement for a bulk node, $\overline{\mathbf{u}}=$ $\overline{\mathbf{L}} \mathbf{U}$;

- $(\delta \widetilde{\mathbf{u}})_{i}=(\widetilde{\mathbf{u}}-\overline{\mathbf{u}})_{i}$ : difference in displacements between the skin nodes and bulk ones;

- $(\widetilde{\mathbf{f}})_{i}=(\widetilde{\mathbf{k}})_{i}(\delta \widetilde{\mathbf{u}})_{i}$ : normal force applied on the bulk.

The choice of $\widetilde{\mathbf{k}}$ and $\widetilde{\mathbf{l}}_{0}$ is discussed later. With these notations, the skin problem equations are summarized as:

$$
\widetilde{\mathbf{f}}=\widetilde{\mathbf{k}} \circ \delta \widetilde{\mathbf{u}}
$$

With time-integration performed by CD-Lagrange scheme as for 1D case, the discrete dynamics equations of bulk and skin are:

$$
\begin{aligned}
& \mathbf{U}_{n+1}=\mathbf{U}_{n}+h \dot{\mathbf{U}}_{n+\frac{1}{2}} \\
& \mathbf{M}\left(\dot{\mathbf{U}}_{n+\frac{3}{2}}-\dot{\mathbf{U}}_{n+\frac{1}{2}}\right)=h \mathbf{F}\left(\mathbf{U}_{n+1}\right)+\overline{\mathbf{L}}^{t} \widetilde{\mathbf{r}}_{n+\frac{3}{2}} \\
& \widetilde{\mathbf{u}}_{n+1}=\widetilde{\mathbf{u}}_{n}+h \dot{\widetilde{\mathbf{u}}}_{n+\frac{1}{2}} \\
& \widetilde{\mathbf{r}}_{n+\frac{3}{2}}=h \widetilde{\mathbf{k}} \circ \delta \widetilde{\mathbf{u}}_{n+1}
\end{aligned}
$$

The Equations (27), 29) are the time-integration relations. And the Equations 28, (30) are the dynamic equations for bulk and skin respectively. As in $1 \mathrm{D}$, the skin contact nodes have no inertia. Their velocities are given by a similar local contact law to the $1 \mathrm{D}$ case:

$$
\begin{aligned}
& \forall i \in \Gamma_{b}, \\
& \text { If }\left(\mathbf{g}_{n+1}\right)_{i}>0 \text {, } \\
& \left(\dot{\widetilde{\mathbf{u}}}_{n+\frac{3}{2}}\right)_{i}=\left(\dot{\overline{\mathbf{u}}}_{n+\frac{3}{2}}^{\text {free }}\right)_{i} \\
& \left\{\begin{aligned}
\text { If }\left(\widetilde{\mathbf{r}}_{n+\frac{3}{2}}\right)_{i} \geqslant 0, & \left(\dot{\widetilde{\mathbf{u}}}_{n+\frac{3}{2}}\right)_{i}=0 \\
\text { Else }\left(\widetilde{\mathbf{r}}_{n+\frac{3}{2}}\right)_{i}<0, & \left(\dot{\widetilde{\mathbf{u}}}_{n+\frac{3}{2}}\right)_{i}=\left(\dot{\overline{\mathbf{u}}}_{n+\frac{3}{2}}^{\text {free }}\right)_{i}^{+}
\end{aligned}\right.
\end{aligned}
$$

where $\dot{\mathbf{U}}_{n+\frac{3}{2}}^{\text {free }}$ is the free bulk velocity without skin action:

$$
\dot{\mathbf{U}}_{n+\frac{3}{2}}^{\text {free }}=\dot{\mathbf{U}}_{n+\frac{1}{2}}+h \mathbf{M}^{-1} \mathbf{F}\left(\mathbf{U}_{\mathbf{n}+\mathbf{1}}\right)
$$

and $\dot{\overline{\mathbf{u}}}_{n+\frac{3}{2}}^{\text {free }}$ is its projection on $\Gamma_{b}$ according to $\boldsymbol{n}$ :

$$
\dot{\overline{\mathbf{u}}}_{n+\frac{3}{2}}^{\text {free }}=\overline{\mathbf{L}}_{n+\frac{3}{2}}^{\text {free }}
$$

The gap $\mathbf{g}_{N}$ is here the distance between nodes of $\Gamma_{b}$ and $\Gamma_{r}$ along $\boldsymbol{- n}$. This choice leads to a clear definition of contact normal: $\boldsymbol{n}$ matches the outer normal of $\Gamma_{r}$ when contact happens, i.e. $\mathbf{g}_{N}=0$. But the persistency condition is not applied on $\Gamma_{b}$, but on the massless nodes. The persistency condition is then not enforced on $\Gamma_{b}$ : the penetration is nonconstant during contact, and the normal velocities are not equal to zero. $\Gamma_{c}$ has no physical sense here as the skin is only a set of numerical paremeters: $\delta \widetilde{\mathbf{u}}$ and $\widetilde{\mathbf{k}}$. Doing this, $\widetilde{\mathbf{l}}_{0}$ is definitely no more necessary.

This formulation, with a totally numerical massless skin, is close to a penalty one for the nodes on $\Gamma_{b}$. Indeed a force is applied on $\Gamma_{b}$ depending on penetration $\delta \widetilde{\mathbf{u}}$ and a numerical multiplication coefficient $\widetilde{\mathbf{k}}$. The main difference is that the skin elements stay active after release.

The algorithm described in Algorithm 1 is still valid, as the energy balance of Equation 23. But it is difficult to cancel the energy loss after release in $3 \mathrm{D}$. The "release time" is no more unique, but exists for each normal elements. As a reminder to obtain a conservative energy balance, each $(\delta \widetilde{\mathbf{u}})_{i}$ must be equal to zero on a discrete time when it releases. This is 


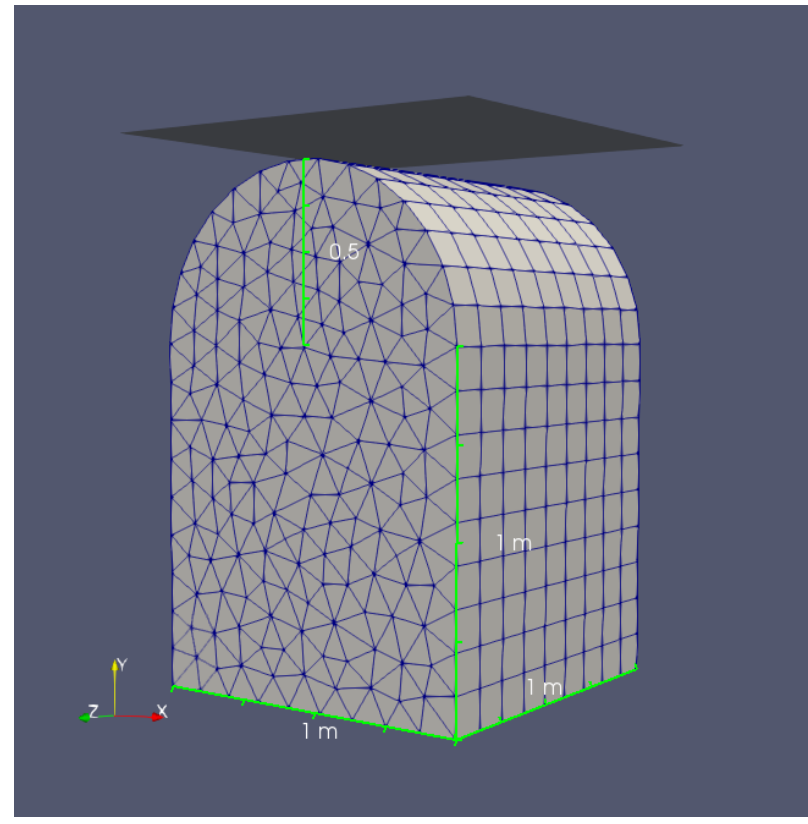

Figure 15: Impacting dome - Initial mesh and geometry

theoretically possible by adjusting $\widetilde{\mathbf{k}}$, but highly difficult in practice. It remains to do a detailed study of the influence of $\widetilde{\mathbf{k}}$ in 3D on the energy balance. But this extra numerical parameter provides a way to improve it without any major change on the solution.

\subsection{Numerical results on impacting dome}

This scheme is used on the case of impacting dome depicted on Figure 15 and set with the values in Table 3 . The dome has an initial uniform velocity along $\boldsymbol{y}$. The upper nodes of the cylindrical part forming $\Gamma_{b}$. Normal massless skin elements are added on them. The assumption is made that the inner contact normal is along $-\boldsymbol{y}$. This seems valid for the line of nodes at the top (along $\boldsymbol{z}$ ), and two closest lines.

This does not correspond to a physical case: the linear elastic material law is used for large deformations. The purpose is to illustrate the ability of contact formulation. The initial velocity $\mathbf{v}_{0}$ is along $\mathbf{y}$.

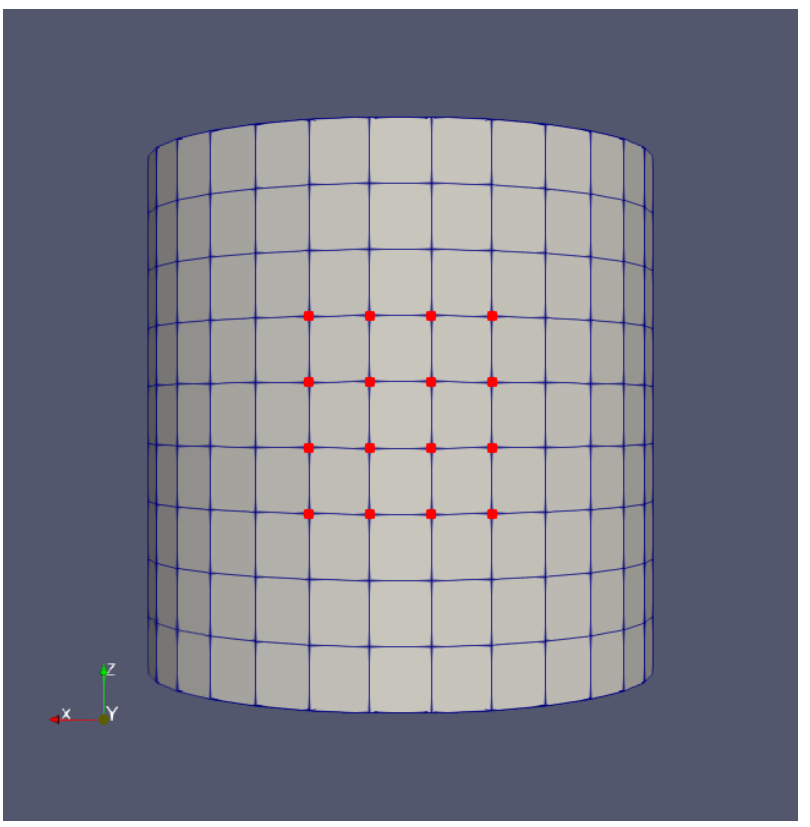

Figure 16: Impacting dome - Central contact zone

\begin{tabular}{ll}
\hline Parameters & Values \\
\hline density $\rho$ & $2000 \mathrm{~kg} \cdot \mathrm{m}^{-3}$ \\
Young modulus $E$ & $1 \times 10^{7} \mathrm{~Pa}$ \\
Poisson's ratio $\nu$ & 0.3 \\
initial velocity $v_{0}$ & $5 \mathrm{~m} \cdot \mathrm{s}^{-1}$ \\
\hline characteristic mesh length $l_{e}$ & $1 / 9 \mathrm{~m}$ \\
critical time step $d t_{\mathrm{CFL}}$ & $8.55 \times 10^{-4} \mathrm{~s}$ \\
time step $h$ & $7.69 \times 10^{-4} \mathrm{~s}$ \\
skin rigidity $\widetilde{\mathbf{k}}$ & $1.91 \times 10^{6} \mathrm{~N} \cdot \mathrm{m}^{-1}$ \\
distance between dome and & $0.102 \mathrm{~m}$ \\
plane $h_{0}$ & \\
\hline
\end{tabular}

Table 3: Numerical values of the 3D impacting dome 


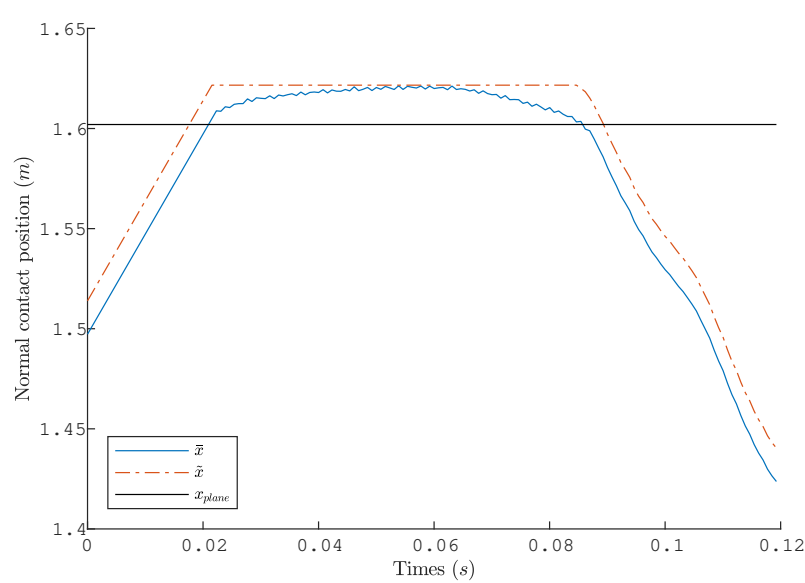

Figure 17: Normal contact position - Mean value on central contact zone

Here the rigidity of normal massless elements is equal to $\widetilde{\mathbf{k}}$ for nodes inside the dome, and $\widetilde{\mathbf{k}} / 2$ for nodes on edges of the dome (as the nodes belong only to two elements). $\widetilde{\mathbf{k}}$ is the maximal diagonal value of $\overline{\mathbf{L}} \mathbf{K} \overline{\mathbf{L}}^{t}$, with $\mathbf{K}$ the rigidity matrix (see Table 3 ).

In order to analyse the numerical results, a central contact zone is defined by the nodes in red on figure 16. This zone is used to mean the contact values (velocities, displacements, impulses). For positions and velocities, the mean is computed only on the first impacting nodes. On Figure 16, this corresponds to the two central lines of nodes on the red zone. The mean node impulse is evaluated over the whole zone. It integrates weighting coefficients depending on position in the zone: if the node is on a corner, the coefficient is $1 / 4$; if the node is on an edge, it is $1 / 2$; and inside, it is 1 . These quantities are depicted in the following graphs.

On Figure 17, the mean position of $\Gamma_{b}$ nodes is denoted by $\bar{x}$. The penetration is directly visible as the difference between $x_{\text {plane }}$ and $\bar{x}$. It changes during the contact because the node is not stopped. As mentioned before, the persistency condition is indeed set on massless nodes. The curve $\widetilde{x}$ represents the mean position of numerical massless contact nodes (with an arbitrary $\widetilde{\mathbf{l}}_{0}$ ). They do not move during contact, respecting the persistency contact constraint. The

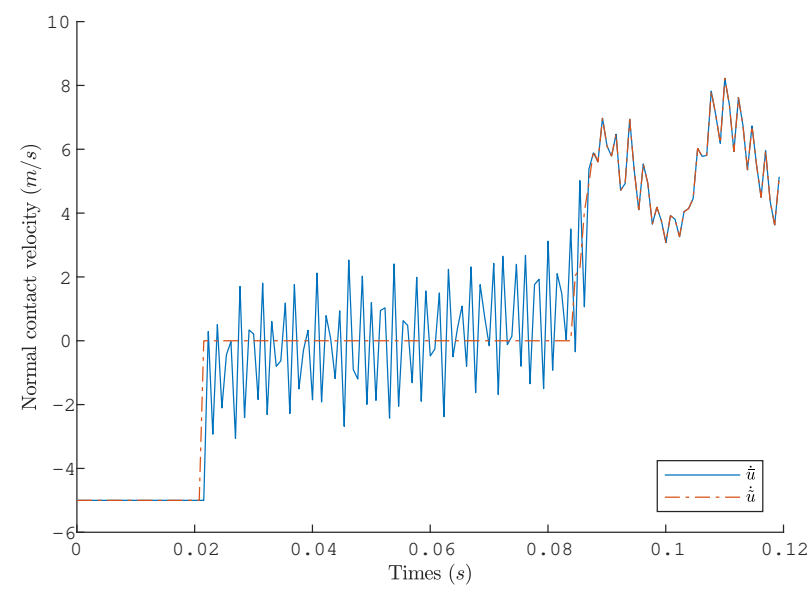

Figure 18: Normal contact velocities - Mean value on central contact zone

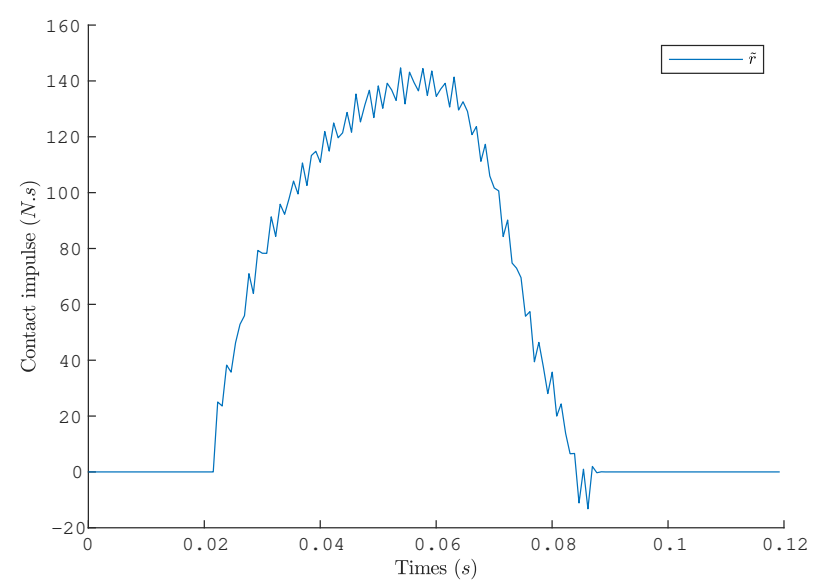

Figure 19: Contact impulse - Weighted value on central contact zone 


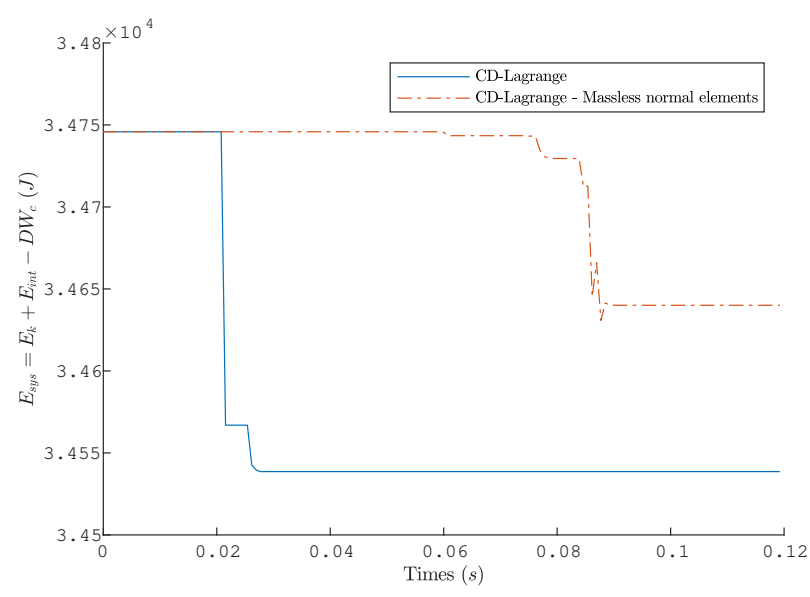

Figure 20: System energies for CD-Lagrange with and without normal massless elements

same pattern happens on mean velocities represented on Figure 18. The velocities of massless contact nodes are equal to zero. But the velocities of $\Gamma_{b}$ nodes are oscillating. These oscillations are also visible on the contact impulse on Figure 19

Despite the non-constant penetration and these oscillations, the energy balance is better than for the reference CD-Lagrange with consistent mass matrix. Figure 20 shows the energy balances for both: the total loss of energy is $0.3 \%$ of the initial energy for the CD-Lagrange with normal massless elements, and $0.6 \%$ for the reference CD-Lagrange. If the two energy losses are low, the gain in energy loss is of $50 \%$. Moreover only one bounce is simulated here, and the number of contact DOFs is low in comparison with the total number of DOFs. In case of multiple bounces or for a larger contact boundary the energy loss will be higher, and the gain even more interesting. This gain is obtained without any optimization in the choice of $\widetilde{\mathbf{k}}$, which shows the scheme ability to improve the energy balance.

The Figures 21, 22, 23, 24 represents the deformed mesh for several times colored by $\left(\overline{\mathbf{L}}^{t} \widetilde{\mathbf{r}}\right) \cdot \boldsymbol{y}$ :

- at impact on figure 21 .

- during contact, when the contact impulse is maximal, on figure 22

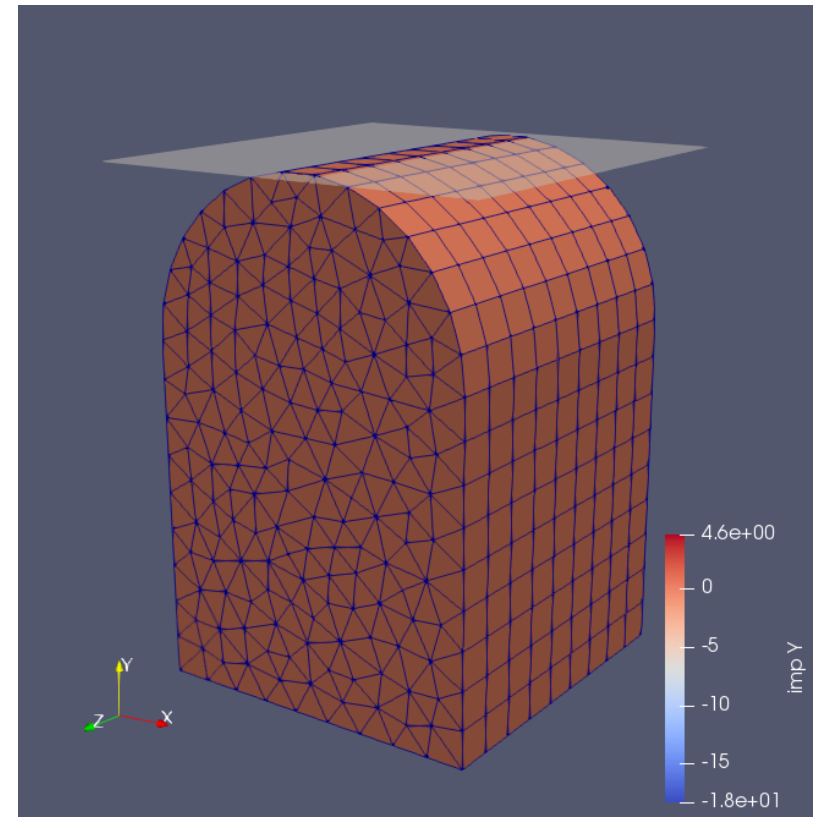

Figure 21: Deformed mesh - Impact

- at release on figure 23

- after release on figure 24.

The space-time convergence is numerically checked on the dome test case. For each space-time refinement, two numerical solutions are computed: one as a reference by classical CD-Lagrange scheme, and one by the CD-Lagrange with normal massless elements. For each solution, the displacement of $\Gamma_{b}$ nodes and contact impulse are averaged on central contact zone. If the number of nodes in it changes, its geometrical dimensions stay the same. The error is defined as the graph distance between the reference solution and the normal massless elements one with the norm described by Equation (24). The reference CD-Lagrange scheme is supposed to converge, as mentioned in [15, 13. The mesh of impacting dome is recomputed with five mesh lengths: $1 / 3 \mathrm{~m}, 1 / 6 \mathrm{~m}, 1 / 9 \mathrm{~m}$, $1 / 12 \mathrm{~m}, 1 / 15 \mathrm{~m}$. For each mesh size, the time-step is set at $0.9 \times d t_{\mathrm{CFL}}$. The results are represented on Figures 25, 26. The rate of convergence is close to order 1 as in the $1 \mathrm{D}$ case. 


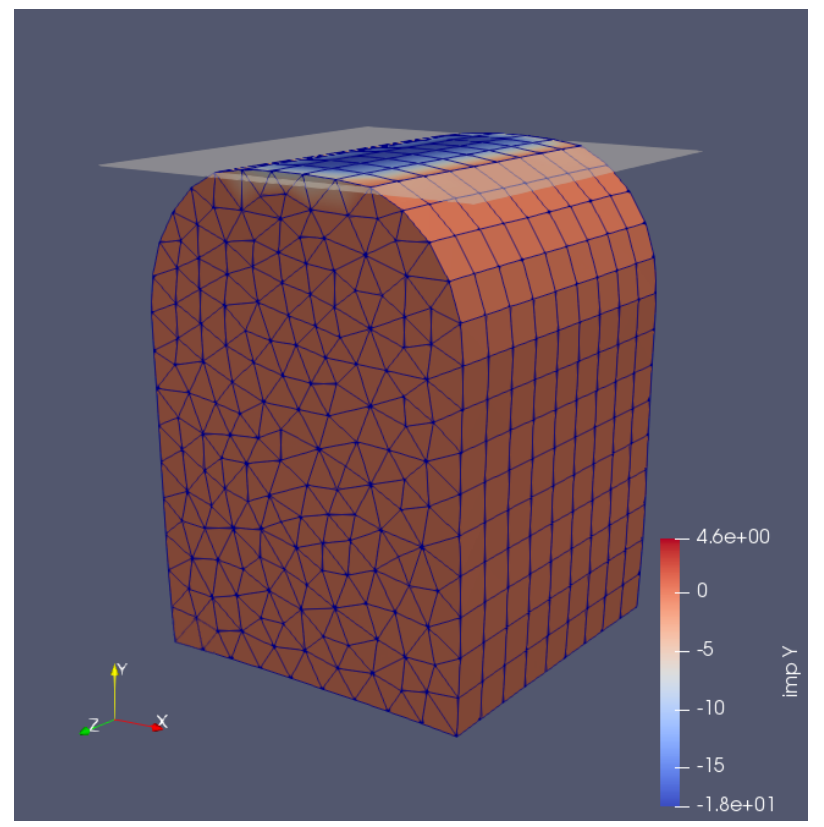

Figure 22: Deformed mesh - Contact

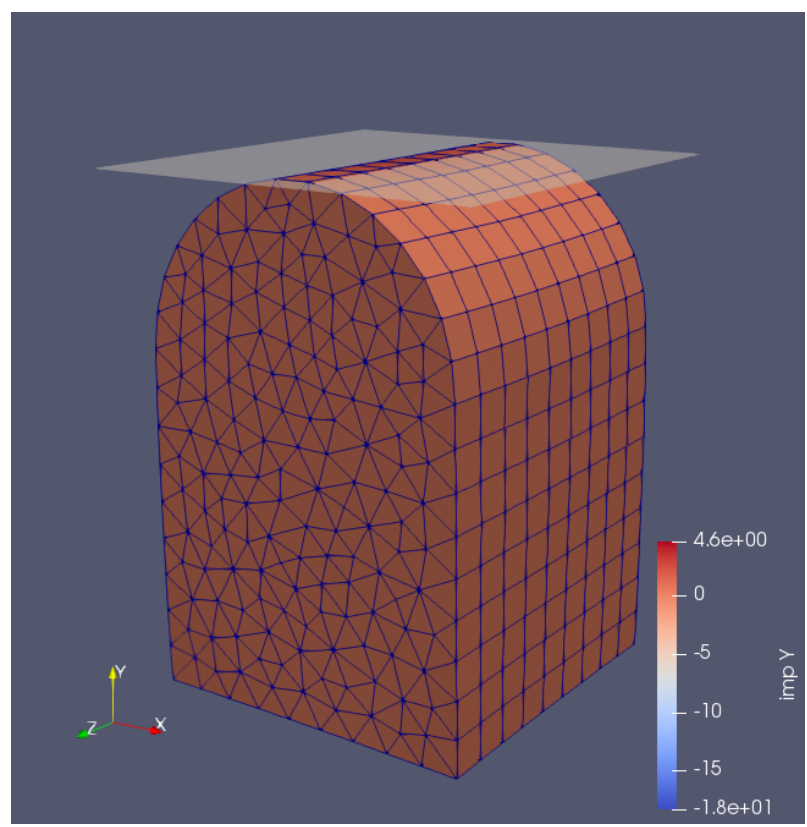

Figure 23: Deformed mesh - Release

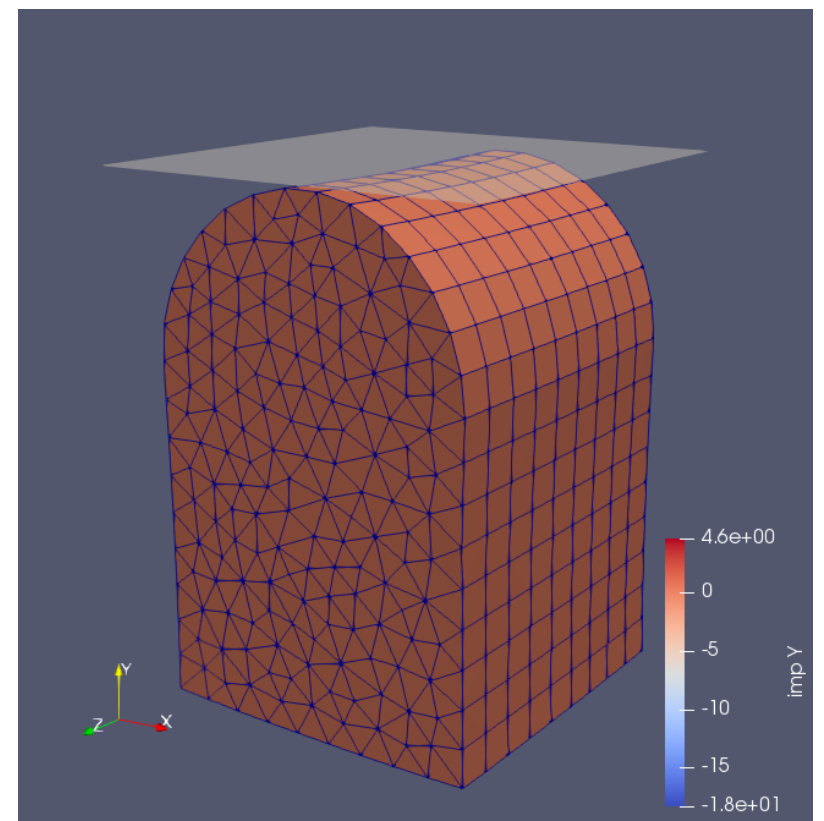

Figure 24: Deformed mesh - After release

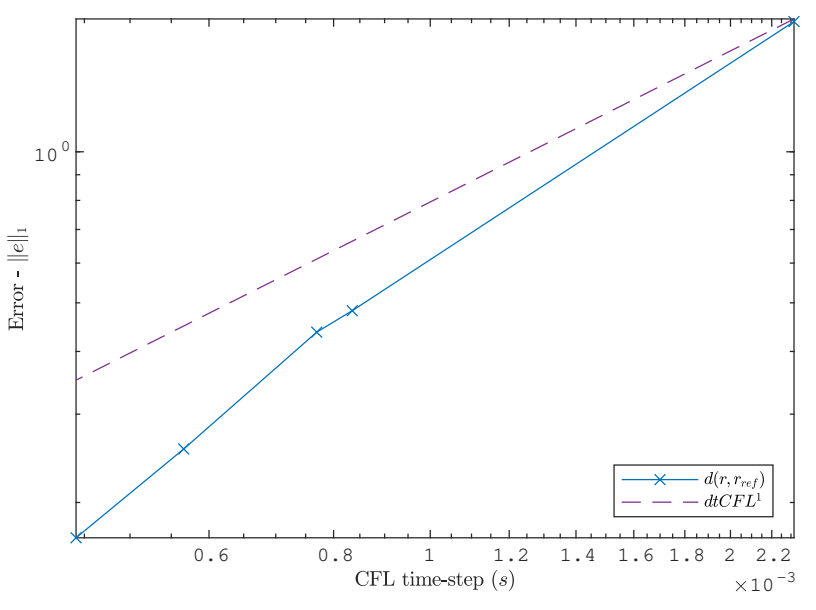

Figure 25: Error - Displacement averaged on central contact zone 


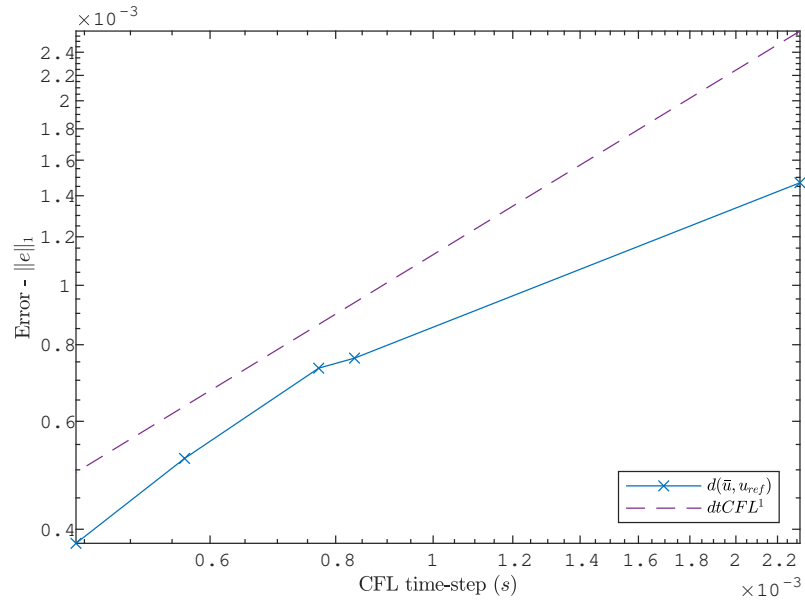

Figure 26: Error - Contact impulse weighted on central contact zone

\subsection{Conclusions on 3D case}

The presented extension from 1D to 3D gives a formulation close from a penalization method. Indeed the nodes on contact boundary (mass ones) check only approximately the velocity contact condition. But the normal massless elements stay active after release. This formulation leads to an accurate energy balance, better than the one of consistent CD-Lagrange. The rigidities of massless skin elements provides numerical parameters which could optimize the energy balance, or the skin response.

The scheme does not present extra difficulties for implementation compared to classic schemes. It consists only in adding DOFs (massless contact nodes) on contact boundary with an associated rigidity. It does not depend on the material law, and is fully compatible with large deformations.

\section{Conclusions}

The singular mass method brings new properties into the CD-Lagrange scheme. For the 1D case, it can turn into an energy conserving scheme which is new in the explicit framework. In order to keep a contact constraint on velocity, a contact law is proposed. It determines the velocity of massless contact node ac- cording to a persistency condition. The proposed 3D extension gives a kind of penalty formulation but for a contact constraint on velocity. It introduces for each contact node a numerical parameter: the rigidity of massless element. The energy balance is better than for the reference CD-Lagrange. And moreover this new formulation is easily implementable, being fully compatible with large deformations and non-linear material laws.

The extension of 1D formulation to 3D could be achieved in different manners. It would be interesting to design a 3D singular mass formulation without normal massless elements, but directly by setting massless DOFs in the initial 3D mesh. The major issue is then to compute the velocities of massless DOFs because they are no more determined by the dynamics. This could be done by adapting the contact law.

A direct perspective is to add friction on the 3D case. And in order to fully address industrial formulations, the scheme needs to deal with deformable-deformable, or rigid-deformable contact problems with non conforming meshes at the interface. This could be done by mortar methods [37, 36, 35.

\section{Appendix: a parallel between the contact law and the velocity contact condition of Moreau- Jean}

The contact law described by Equations (10), (11) is close from the Moreau-Jean velocity conditions [27, 20]. With a mass contact node, these conditions express as:

$$
\begin{array}{ll}
\text { If } \mathbf{g}_{n+1}>0, & \widetilde{\mathbf{r}}_{n+\frac{3}{2}}=0 \\
\text { If } \mathbf{g}_{n+1} \leqslant 0, & 0 \leqslant \widetilde{\mathbf{r}}_{n+\frac{3}{2}} \perp \dot{\widetilde{\mathbf{u}}}_{n+\frac{3}{2}} \geqslant 0
\end{array}
$$

The Equation (36) describes the free of contact state, and the Equation (37) describes the active contact. $\widetilde{\mathbf{r}}_{n+\frac{3}{2}}$, the contact impulse, acts as a Lagrange multiplier. It imposes $\dot{\widetilde{\mathbf{u}}}_{n+\frac{3}{2}}$, the contact node velocity, if it does not respect the contact conditions: 
- If $\dot{\widetilde{\mathbf{u}}}_{n+\frac{3}{2}} \leqslant 0$, the beam tends to penetrate into rigid frontier. The dynamic gives a $\widetilde{\mathbf{r}}_{n+\frac{3}{2}}>0$ to stop the contact node. It compensates both the internal stress of skin and the inertia of contact node. The skin is in compression.

- If $\dot{\widetilde{\mathbf{u}}}_{n+\frac{3}{2}}>0$, the beam tends to leave the rigid frontier. $\widetilde{\mathbf{r}}_{n+\frac{3}{2}}$ is set to zero because the system already meets the contact conditions. The skin is no more constrained.

The velocity contact law (10), (11) for singular mass matrix follows the same principles as the Moreau-Jean's condition (36), (37). But the MoreauJean's condition have access to $\widetilde{\mathbf{u}}_{n+\frac{3}{2}}$ through the dynamic before to set $\widetilde{\mathbf{r}}_{n+\frac{3}{2}}$. At the release time-step, $\dot{\widetilde{\mathbf{u}}}_{n+\frac{3}{2}}>0$ and so $\widetilde{\mathbf{r}}_{n+\frac{3}{2}}$ is directly set to zero. For the velocity contact law, $\dot{\widetilde{\mathbf{u}}}_{n+\frac{3}{2}}$ is computed once $\widetilde{\mathbf{r}}_{n+\frac{3}{2}}$ is known. This leads to a release "late" of one timestep: at release, $\widetilde{\mathbf{r}}_{n+\frac{3}{2}} \leqslant 0$ but already fixed and $\dot{\widetilde{\mathbf{u}}}_{n+\frac{3}{2}}>0$. This temporary violation on positivity of contact stresses is necessary as no dynamical link exists between $\widetilde{\mathbf{r}}_{n+\frac{3}{2}}$ and $\dot{\widetilde{\mathbf{u}}}_{n+\frac{3}{2}}$.

\section{Declarations}

\section{Funding}

We gratefully acknowledge the French National Association for Research and Technology (ANRT, CIFRE grant number 2017/1555). This work was supported by the "Manufacture Française de Pneumatiques Michelin".

\section{Conflict of interest}

The authors declare that they have no conflict of interest.

\section{Code availability}

All computations were done with a custom code.

\section{References}

[1] Acary V (2012) Higher order event capturing time-stepping schemes for nonsmooth multibody systems with unilateral constraints and impacts. Applied Numerical Mathematics 62(10):12591275, DOI 10.1016/j.apnum.2012.06.026

[2] Belytschko T, Neal MO (1991) Contact-impact by the pinball algorithm with penalty and Lagrangian methods. International Journal for $\mathrm{Nu}-$ merical Methods in Engineering 31(3):547-572, DOI 10.1002/nme.1620310309

[3] Belytschko T, Liu WK, Moran B, Elkhodary KI (2014) Nonlinear Finite Elements For Continua And Structures, 2nd edn. Wiley, Chichester, West Sussex, United Kingdom

[4] Brüls O, Acary V, Cardona A (2014) Simultaneous enforcement of constraints at position and velocity levels in the nonsmooth generalized- $\alpha$ scheme. Computer Methods in Applied Mechanics and Engineering 281(1):131-161, DOI 10.1016/j.cma.2014.07.025

[5] Carpenter NJ, Taylor RL, Katona MG (1991) Lagrange constraints for transient finite element surface contact. International Journal for $\mathrm{Nu}$ merical Methods in Engineering 32(1):103-128, DOI 10.1002/nme.1620320107

[6] Chen QZ, Acary V, Virlez G, Brüls O (2013) A nonsmooth generalized- $\alpha$ scheme for flexible multibody systems with unilateral constraints. International Journal for Numerical Methods in Engineering 96(8):487-511, DOI 10.1002/nme. 4563

[7] Chouly F, Fabre M, Hild P, Mlika R, Pousin J, Renard Y (2016) An overview of recent results on Nitsche's method for contact problems. In: S B, E B, M L, M O (eds) UCL Workshop 2016, UCL (University College London), Springer, London, United Kingdom, Geometrically Unfitted Finite Element Methods and Applications, vol 121, pp 93-141, DOI 10.1007/978-3-319-71431-8\_4, URL https:// hal.archives-ouvertes.fr/hal-01403003 
[8] Chung J, Hulbert GM (1993) A Time Integration Algorithm for Structural Dynamics With Improved Numerical Dissipation: The Generalized- $\alpha$ Method. Journal of Applied Mechanics 60(2):371-375, DOI 10.1115/1. 2900803

[9] Cirak F, West M (2005) Decomposition contact response (DCR) for explicit finite element dynamics. International Journal for Numerical Methods in Engineering 64(8):1078-1110, DOI 10.1002/nme. 1400

[10] Curnier A (1999) Unilateral Contact. In: Wriggers P, Panagiotopoulos P (eds) New Developments in Contact Problems, Springer Vienna, Vienna, chap Chapter 1, pp 1-54, DOI 10.1007/ 978-3-7091-2496-3

[11] Dabaghi F, Petrov A, Pousin J, Renard Y (2016) A robust finite element redistribution approach for elastodynamic contact problems. Applied Numerical Mathematics 103:48-71, DOI 10.1016/j.apnum.2015.12.004

[12] Dabaghi F, Krejčí P, Petrov A, Pousin J, Renard Y (2019) A weighted finite element mass redistribution method for dynamic contact problems. Journal of Computational and Applied Mathematics 345:338-356, DOI 10.1016/j.cam.2018.06. 030

[13] Di Stasio J, Dureisseix D, Gravouil A, Georges G, Homolle T (2019) Benchmark cases for robust explicit time integrators in non-smooth transient dynamics. Advanced Modeling and Simulation in Engineering Sciences 6(2):0, DOI 10.1186/s40323-019-0126-y

[14] Doyen D, Ern A, Piperno S (2011) TimeIntegration Schemes for the Finite Element Dynamic Signorini Problem. SIAM Journal on Scientific Computing 33(1):223-249, DOI 10.1137/ 100791440

[15] Fekak FE, Brun M, Gravouil A, Depale B (2017) A new heterogeneous asynchronous explicit-implicit time integrator for nonsmooth dy- namics. Computational Mechanics 60(1):1-21, DOI 10.1007/s00466-017-1397-0

[16] Fetecau RC, Marsden JE, Ortiz M, West M (2003) Nonsmooth Lagrangian Mechanics and Variational Collision Integrators. SIAM Journal on Applied Dynamical Systems 2(3):381-416, DOI 10.1137/S1111111102406038

[17] Hager C, Hüeber S, Wohlmuth BI (2007) A stable energy-conserving approach for frictional contact problems based on quadrature formulas. International Journal for Numerical Methods in Engineering 73(2):205-225, DOI 10.1002/nme. 2069

[18] Hauret P (2010) Mixed interpretation and extensions of the equivalent mass matrix approach for elastodynamics with contact. Computer Methods in Applied Mechanics and Engineering 199(45-48):2941-2957, DOI 10.1016/j. cma.2010.06.004

[19] Hilber HM, Hughes TJ, Taylor RL (1977) Improved numerical dissipation for time integration algorithms in structural dynamics. Earthquake Engineering \& Structural Dynamics 5(3):283292, DOI 10.1002/eqe.4290050306

[20] Jean M (1999) The non-smooth contact dynamics method. Computer Methods in Applied Mechanics and Engineering 177(3-4):235-257, DOI 10.1016/S0045-7825(98)00383-1

[21] Kaufman DM, Pai DK (2012) Geometric Numerical Integration of Inequality Constrained, Nonsmooth Hamiltonian Systems. SIAM Journal on Scientific Computing 34(5):A2670 A2703, DOI 10.1137/100800105, 1007.2233

[22] Khenous HB, Laborde P, Renard Y (2008) Mass redistribution method for finite element contact problems in elastodynamics. European Journal of Mechanics, A/Solids 27(5):918-932, DOI 10. 1016/j.euromechsol.2008.01.001

[23] Krause R, Walloth M (2012) Presentation and comparison of selected algorithms for dynamic contact based on the Newmark scheme. Applied 
Numerical Mathematics 62(10):1393-1410, DOI 10.1016/j.apnum.2012.06.014

[24] Krenk S (2006) Energy conservation in Newmark based time integration algorithms. Computer Methods in Applied Mechanics and Engineering 195(44-47):6110-6124, DOI 10.1016/j. cma.2005.12.001

[25] Laursen TA, Chawla V (1997) Design of energy conserving algorithms for frictionless dynamic contact problems. International Journal for Numerical Methods in Engineering 40(5):863886, DOI 10.1002/(SICI)1097-0207(19970315) 40:5<863::AID-NME92 $>3.0 . \mathrm{CO} ; 2-\mathrm{V}$

[26] Marsden JE, West M (2001) Discrete mechanics and variational integrators. Acta Numerica 2001 10:357-514, DOI 10.1017/S096249290100006X

[27] Moreau JJ (1999) Numerical aspects of the sweeping process. Computer Methods in Applied Mechanics and Engineering 177(3-4):329349, DOI 10.1016/S0045-7825(98)00387-9

[28] Newmark NM (1959) A Method of Computation for Structural Dynamics. Journal of the Engineering Mechanics Division 85(7):67-94

[29] Paoli L, Schatzman M (2002) A Numerical Scheme for Impact Problems I: The Onedimensional Case. SIAM Journal on Numerical Analysis 40(2):702-733, DOI 10.1137/ S0036142900378728

[30] Paoli L, Schatzman M (2002) A Numerical Scheme for Impact Problems II: The Multidimensional Case. SIAM Journal on $\mathrm{Nu}-$ merical Analysis 40(2):734-768, DOI 10.1137/ S003614290037873X

[31] Renard Y (2010) The singular dynamic method for constrained second order hyperbolic equations: Application to dynamic contact problems. Journal of Computational and Applied Mathematics 234(3):906-923, DOI 10.1016/j.cam.2010. 01.058
[32] Simo JC, Tarnow N (1992) The discrete energymomentum method. Conserving algorithms for nonlinear elastodynamics. ZAMP Zeitschrift für angewandte Mathematik und Physik 43(5):757792, DOI 10.1007/BF00913408

[33] Simo JC, Tarnow N, Wong KK (1992) Exact energy-momentum conserving algorithms and symplectic schemes for nonlinear dynamics. Computer Methods in Applied Mechanics and Engineering 100(1):63-116, DOI 10.1016/ 0045-7825(92)90115-Z

[34] Tkachuk A, Wohlmuth BI, Bischoff M (2013) Hybrid-mixed discretization of elasto-dynamic contact problems using consistent singular mass matrices. International Journal for Numerical Methods in Engineering 94(5):473-493, DOI $10.1002 /$ nme. 4457

[35] Wohlmuth B (2011) Variationally consistent discretization schemes and numerical algorithms for contact problems. Acta Numerica 20:569-734, DOI 10.1017/S0962492911000079

[36] Wohlmuth BI (2000) A Mortar Finite Element Method Using Dual Spaces for the Lagrange Multiplier. SIAM Journal on Numerical Analysis 38(3):989-1012, DOI 10.1137/ S0036142999350929

[37] Wriggers P (2006) Computational contact mechanics, 2nd edn. Springer Berlin Heidelberg, Berlin, Heidelberg, DOI 10.1007/978-3-540-32609-0 